\title{
LES TURBINES HYDRAULIQUES QUELQUES PROJETS ET RÉALISATIONS ACTUELS
}

\author{
J. CHENAIS \\ Ancien Elève de l'Ecole Polytechnique \\ Ingénieur E.S.E. et P.T.T.
}

Nous allons essayer dans cet article de mettre en relief quelques-uns des problemes que pose actuellement la technique des turbines hydrauliques et de: donner une idée, dans la mesure où le permettront les quelques chiffres et documents que nous avons pu réunir, des perfectionnements dont bénéficient les grandes réalisations actuellement en cours - ou en projet - et des moyens de plus en plus puissants mis en cuvre pour leur achèvement.

Ce travail se divisera en trois parties.

Après avoir domné quelques chifres relatifs aux installations nouvelles mises en route depuis 1939 en France, nous consacrerons une première partie au réaménagement de quelques centrales anciennes, auxquelles, en quelque sorte, un sang nowveat a élé transfusé; nous parlerons en particulier de Jonage. des usines de la vallée de la Tél, dans les PyrénéesOrientales, enfin de celles de Fond-de-France, dans le massif d'Allevard, et de Champ-sur-Drac, près de Grenoble.

Dans une seconde partie, nous dirons quelques mots de la place que tiennent les systèmes automatiques dans la commande des centrales hydro-électriques et de cette question à la fois si complexe et si intéressante qu'est la régulation des Centrales interconnectées.

Enfin, nous examinerons, dans une troisieme pattie, quelques dispositions caractéristiques des trois principaux types de turbines: hélice à pales orientables, Pelton, Francis, et nous terminerons par une brève description des machines motrices les plus puissantes du monde, parmi lesquelles, au milieu des machines du Boulder Dam, de Shipshaw, de la Sungari et du Grand Conlee Dam, les turbines en construction pour les centrales de Génissiat et de Chaslang, tiennent une place qui fera honneur à la technique française.
Si les terribles années que nous venons de traverser et les difficultés actuelles, encore considérables, ont pesé lourdement et pèsent toujours sur les constructions hydrauliques francaises, nous pouvons cependant trouver un grand réconfort dans l'examen du bilan des travaux effectués dans ce domaine depuis 1940 .

La vie des grands chantices s'est certes trouve ralentie du fait du manque de matières premières, de main-d'œuvre, de transports, mais les grandes réalisations qui sont actuellement en cours marquent incontestablement un pas en avant par rapport à celles d'avant-guerre, en particulier du point de vue de la puissance unitaire des groupes.

Après les six machines de $42.500 \mathrm{CV}$ de Brommal, les quatre de $52.000 \mathrm{CV}$ de Sarrans, sur la Truyère, les quatre de $46.200 \mathrm{CV}$ de Maréges, les six groupes de $16.000 \mathrm{CV}$ du Sautel, les trois turbines de 40.000 $\mathrm{CV}$ de Bissorte qui jalonnent la technique 1930-39, nous avons déjà vu, entre 1939 et 1945 , dans les centrales de grande puissance, la mise en route des deux turbines de $21.750 \mathrm{CV}$ de Pradieres, du groups de $44.000 \mathrm{CV}$ de Fond-de-France, de celui de 55.600 $\mathrm{CV}$ du portillon, des quatre groupes de $16.600 \mathrm{CV}$ de Pont-Escoffier, sur le Vénéon, de Saint-EtienneCantales, sur la Cère, avec trois groupes de 34.000 $\mathrm{CV}$, de L'Aigle (1), sur la Dordogne, avec quatre de $70.000 \mathrm{CV}$, et nous allons prochainement assister a celle de Cordéac, str le Drac, avec deux groupes de $28.000 \mathrm{CV}$ et $56.000 \mathrm{CV}$. Puis suivra Génissiat, aves quatre groupes de $90.000 \mathrm{CV}$ et, plus tard, deux ds $120.000 \mathrm{CV}$, enfin Chastang, sur la Dordogne, avec des machines de $135.000 \mathrm{CV}$.

(1) Les turbines de l'Aigle ont été construites par Schneider
ef Cie. 
A côté des 210.000 CV que représentent les installations importantes de Pradieres, de Fond-de-France, du. Portillon, de Ponl-Escoffier, il convient de ne pas oublier les quelques 200.000 GV que représentent les installations de puissance unitare moindre, mises en rotite depuis 1940 , parmi lesquelles on peut ciler:

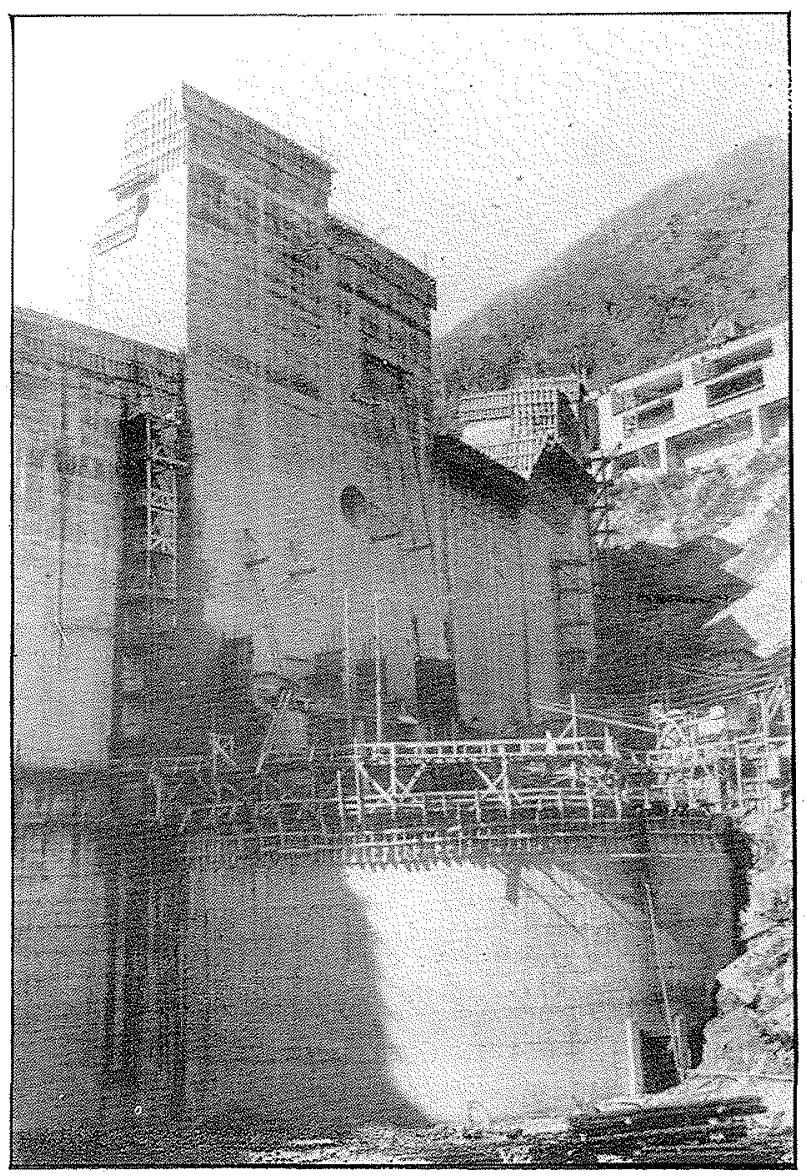

L'AIGLE. - VANNE DE PRISE D'EAU
EN COURS DE MONTAGE (Ensemb!e)

DANS LES ALPES. - Les nouveaux groupes de 4.000 CV. de la Salusaz, celui de Champ-surLrac, les groupes Pelton de $9.500 \mathrm{CV}$. de Ja Rageal et de $4.000 \mathrm{CV}$. de l'Argentiere, la Centrale de calypso basse chute deux groupes de (j.800 CV.), détruile ali cours de la retraite alle. mande en Halrienne el reconstruite depuis.

Massif Central. - Les deux groupes Francis de Monistrol-d Allier $(7.470 \mathrm{CV}$.$) , les turbines hélice$ de linmel.

Poun les Pyrénées. - Les groupes de $6.400 \mathrm{CV}$. de Fonl-Pedrousse, de 12.500 CV. d'Usson, les deux Pelton verticales de la Cassagne, les deux Francis de Saint-Cricq $(6.700 \mathrm{CV}$ ), les six groupes Pelton et Francis de Sonlom, le nouveau groupe de $6.000 \mathrm{CV}$. de Saint-Lary, les trois groupes héliee de $3.470 \mathrm{CV}$. de Baigt, les deux Pelton de 9.000 CV. d'Artigues, la turbine Francis de 5.000 CV. du Teich.

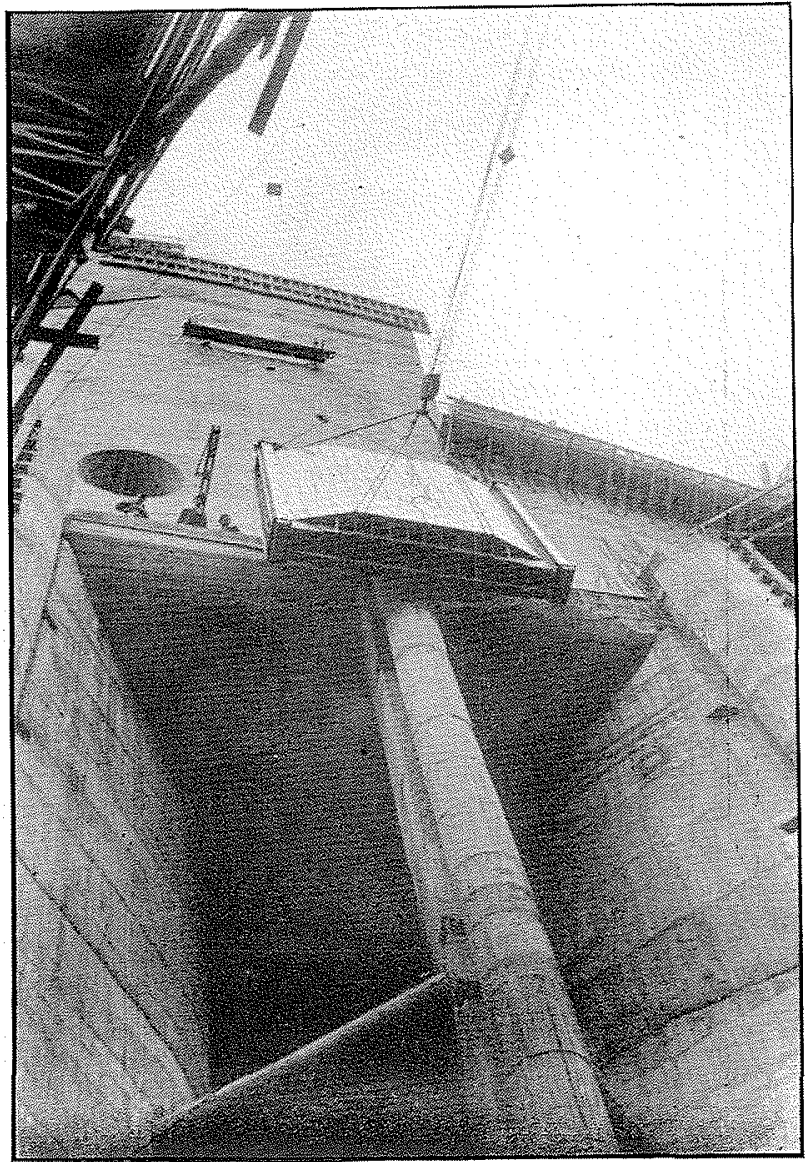

L'AIGLE, - VANNE DE PRISE D'EAU EN COURS DE MONTAGE (Détail)

Cette liste qui est certanement incomplete, ne tient pas compte de Ia production de lefrique du Nord.

l'ensemble de ces machines représente, rien que pour la France métropolitaine, une puissance de l'ordre de $400.000 \mathrm{CV}$., dont l'appoint est heureusement supérieur aux destructions allemandes, qui ont cependant coûté, en Maurienne, plus de $250.000 \mathrm{CS}$ avec Ies Centrales de Pontamajrey, de Saint-Jean, La Praz, Avrieux, de Calypso, et nolis en passons, at surtout de Bissorte. Les destructions de ces Centrales ne sont, évidemment, pas complètes et les travaux que nécessite leur remise en état sont active- 
ment poussés. C'est ainsi que, dans la Centrale de la Bissorle, deux groupes ont repris le service en 1945, et que le troisième a été remis en route en janvier 1946 ; il est à remarquer que, quoique datant ds 1936, ces trois turbines Pelton, qui donnent 40.000 $\mathrm{CV}$ sous $1.160 \mathrm{~m}$., ont élé réinstallées à peu près sans modifications; leur bonno tenue en exploitation, les rendements qu'elles domnent, qui dépassent $90,7 \%$ ne sont, dans le domaine des hautes chutes, guère dépassés aujourd'hui dans le monde, si même ils ont été atteints.

Après ces quelques mots d'introduction, nous allons passer à la description de quelques Centrales
Le nombre encore élevé de modernisations récentes fait d'ailleurs toucher du doigt l'échelle des progrès réalisés depuis l'autre guerre, puisque les dépenses engagées sont compensées:

- d'une part, srâce à l'amélioration du rendement et l'auginentation du débil, dans le cadre d'installations existantes;

- d'autre part, dans cerlains cas, srâce à une plus grande facilite de condute, qui entraine une réduction sensible des frais d'exploitation.

La réalisation-type qui, dans ce domaine, se présente à l'esprit, est certes celle de Jonage. Il con-

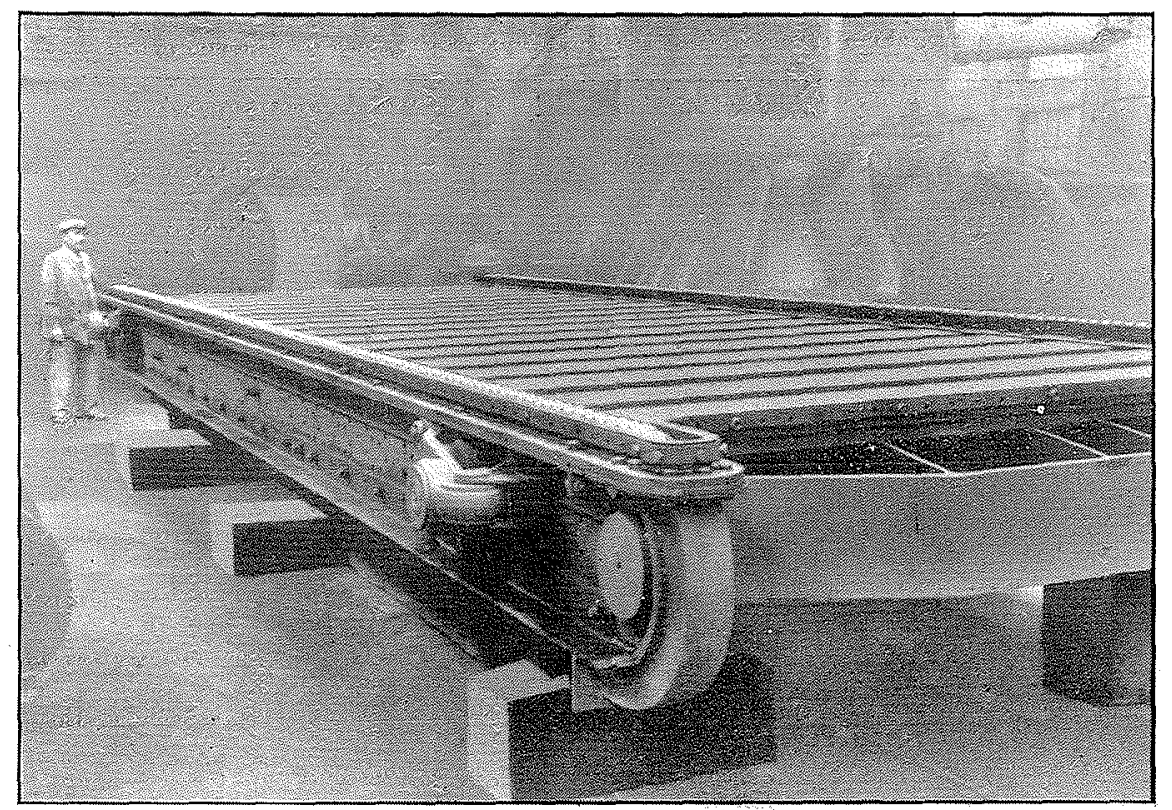

L'AIGLE, - VANNE DE VIDANGE, TYPE "A CHENILLES » POUR PERTUIS DE $2,500 \mathrm{M} . \times 8,500 \mathrm{M}$. HAUTEUR D'EAU SUR LE SEUIL: $75 \mathrm{M}$.

anciennes qui, grâce à des réaménagements récents, fonctionnent maintenant dans des conditions de rendement et avec une facilité de conduite qui n'ont souvent rien à envier à celles des usines les plus modernes.

Ces installations posent aux Ingénieurs des problèmes d'une difficulté particulière, du fait que las dispositions nouvelles doivent s'inscrire dans le car dre des anciemnes; il faut trouver, dans chaque cas, la solution qui assure le gain maximum, en kwh. par an, moyennant la moindre dépense relative. En particulier, le temps d'immobilisation des machines, entre l'arrêt de l'ancienne centrale et la mise en route de la nouvelle, doit être réduit au strict minimum. vient, tout d'abord, de rappeler le bruit qu'avait fait dans le monde, en 1896 , cette construction d'une échelle alors inconnue en Europe, qui mettait en relief l'esprit d'entreprise qu'à si souvent manifesté l'industrie lyonnaise. On peut citer, à ce sujet, l'appréciation de M. Routin qui écrivait à celte époque :

« L'importance de l'entreprise exigeait, pour s:a « mise en cuvre, tous les soins minutieux dont elle « a été l'objet. La station de Jonage peut, en effet, « être comparée comme importance, a la station « fameuse de la Niagara Fall's Company. L'ancien « continent semble enfin se réveiller de sa torpeur « habituelle car, moins de deux ans après la gran« diose tentative américaine, on pourra opposer, en « France, une application aussi audacieuse de l'as« servissement des forces de la nature. » 
Rappelons que la Centrale comportait 16 groupes Jonval et Francis de 1.250-1.350 CV à $120 \mathrm{t} . / \mathrm{m}$., avec des alternateurs triphasés (nouveauté pour l'époque) de 6 metres de diamètre.

Entre 1930 et 1939 , ces groupes anciens, dont un seul a été conservé comme groupe auxiliaire et aussi probablement à titre de témoin du passé, ont fait place à 14 turbines Hélice à pales orientables, da $6.000 \mathrm{CV}$ chacune, à $214 \mathrm{t} . / \mathrm{m}$, sous 12 mètres de chute, ce qui correspond à une vitesse spécifique particulièrement élevée de plus de $700 \mathrm{t}$./m. L'augmentation de chute (à l'origine, elle était de $10 \mathrm{~m}$.) a été obtenue grâce à la construction d'un barrage mobile à cinq pertuis, le barrage de Jons, et la mise en place des nouveaux groupes a nécessité un véri- coups de bélier, essais maintenant historiques, effectués par MN. Camichel, Eydoux et Gariel (1). Les deux chutes qui alimentent cette Centrale ont éte munies, juste avant la récente guerre, l'une de trois turbines Pelton de 3.500 et $7.000 \mathrm{CV}$, construites par Als-Thom Charmilles, l'autre de trois groupes Fran. cis Neyrpic, à axe vertical, de $5.870 \mathrm{CV}$. sous $107 \mathrm{~m}$.

La Centrale de la Cassagne qui occupe le sommet de la vallée de la Tet a été remise en route en 1944 , avec deux turbines Pellon-Neyrpic à axe vertical, de $5.700 \mathrm{CV}$. sous $400 \mathrm{~m}$, de chute. Ces machines remplacent quatre turbines Girard de $2.000 \mathrm{CV}$. La disposition nouvelle, a axe vertical, a permis de réutiliser les maçonneries sans modifications importantes, l'alimentation par en-dessous des turbines

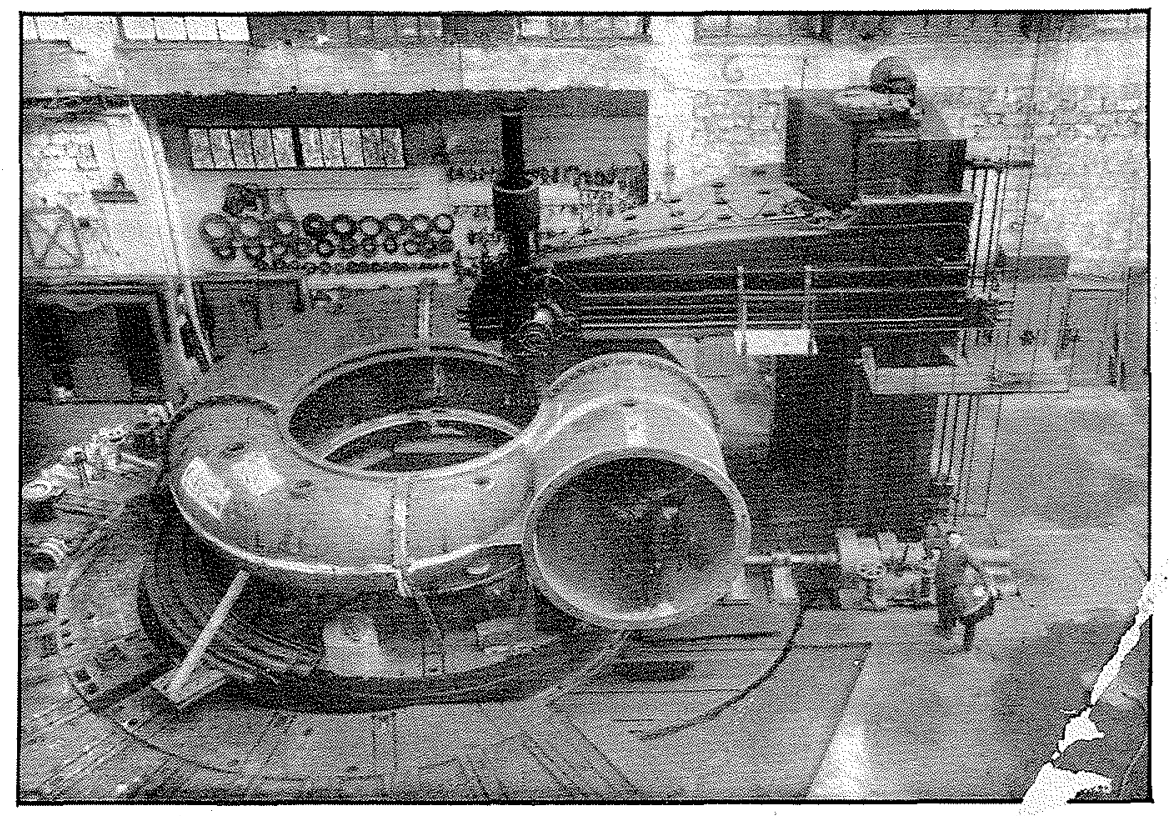

CHAMP-SUR-DRAC. - BACHE SUR TOUR VERTICAL

table tour de force; une partie importante des maconneries a été reprise en sous-cuvre, et cela sans arrêter l'exploitation de l'Usine.

Les turbines ont été fournies par la Socièté Escher-Wyss, par la Société Alsacienne de Constructions Mécaniques, Als-Thom Charmilles, et les Ateliers N.B.P.P.

On jugera de l'ampleur de cette transformation en soulignant que, dans le cadre d'une Centrale de 20.000 CV., en service depuis plus de trente ans, il a été installé un ensemble de turbines pouvant développer plus de $90.000 \mathrm{CV}$.

En passant des Alpes aux Pyrénées, on peut citer, à propos d'usines réaménagées, celles de Soulom qui, en 1916-1917 fût le siège des essais relatifs aux
Girard ayant fait place $\dot{a}$ une alimentation pardessus des turbines Pelton placées à un niveau notablement inférieur : cette disposition permet de grtgner quatre mètres de chute environ. Les quatre anciennes conduites, complètement usées, ont fait place à deux conduites neuves, capables d'un débit supérieur et les quelque $76 \%$ de rendement des turbines Girard, aux $89,8 \%$ des Pelton, si bien que la puissance de la Centrale a pu être portée de 8.000 à 11.400 CV., soit une augmentation de plus de $40 \%$.

Cette centrale est la première d'une série d'installations montées en cascade sux la vallée de la

(1) Camichel, Eydoux et Gariel: " Etude théorique et expérimentale sur les coups de bélier dans les conduites forcées" (Toulouse, Privat, 1919). 
Tet, et destinées à alimenter le réseau de traction de la S.N.C.F.

Immédiatement à l'aval de la Cassagne, la Centrale de Fontpedrouse a été mise en route en 1942, dans le même bâtiment qui abritait, depuis 1913, deux turbines Pelton de $2.200 \mathrm{CV}$. Le canal d'ame- d'altitude des Sepl-Lanx, situés au pied du RocherBlanc, bien connu des skieurs. En 1941, un nouveau battiment fut construit sur l'ancien et une nouvelle conduite forcée fut mise en place. Un nouvean lac du groupe des Sept-Laux fut percé et 'laugmentation de débit réalisée permit de porter la puissance de

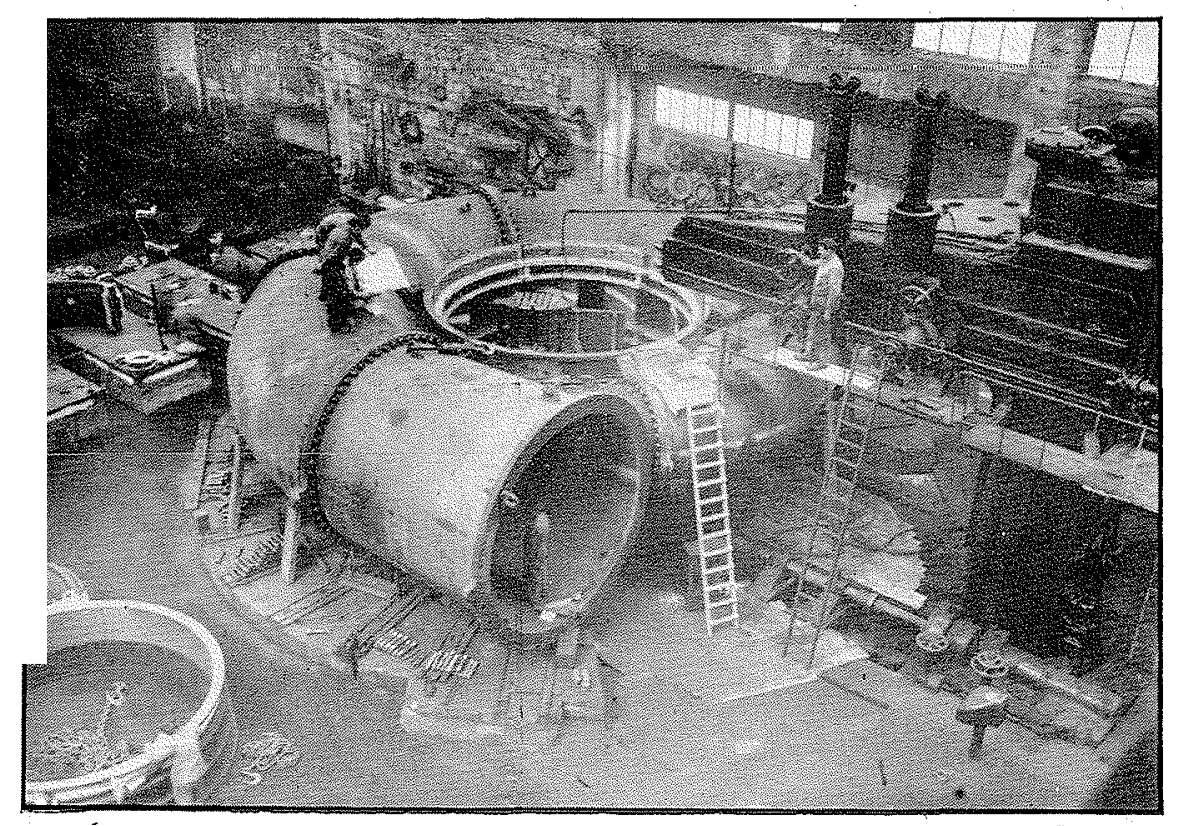

CORDEAC. - BACHE SUR TOUR VERTICAL

née a été remis à neuf : dans certaines sections son tracé a été modifie afin d'éviter des zones de terrain instable. La conduitt forcée a été changée; le débit maximum utilisé est ainsi passé de $2.000 \mathrm{l}$./sec. à $3.800 \mathrm{l} . / \mathrm{sec}$. et le groupe Pelton unique, à marche entièrement automati ${ }_{4}$. qui remplace les anciennes machines, développe $7.400 \mathrm{CV}$ à $428 \mathrm{t}$. $/ \mathrm{min}$. sous $166 \mathrm{~m}$. de chute. La faible hauteur de chute a imposé la disposition à deux rones, quatre jels, qui permet d'utiliser à bon rendement, même de faibles débits d'étiage. A l'aval de la Centrale de Fontpedrouse, deux Centrales nouvelles sont en constructions: celle de Thues qui comportera deux groupes Francis de $4.500 \mathrm{CV}$ sous $75 \mathrm{~m}$. et celle d'Olette ou seront installés deux groupes Pelton à deux roues, matre jets, de $6.800 \mathrm{CV}$ sous $214 \mathrm{~m}$.

Coujours dans le domaine des turbines Pelton, une lisation des plus intéressantes est celle de la ntrale de Fond-de-France. L'ancienne Centrale, ustruite par la Société Générale de Force et Luère, à la fin de la guerre 1914-1918 comportait groupe de $8.000 \mathrm{CV}$ et alimentait à l'origine le réseau continu série. La Centrale prend son eau sous une chute de $1.000 \mathrm{~m}$. environ, dans les lacs la Centrale à $44.000 \mathrm{CV}$ grâce à un groupe Pelton Alsthom-Charmilles à deux roves, deux jets, tournant a $500 \mathrm{t}$./min. Les deux roues sont montées en porte-

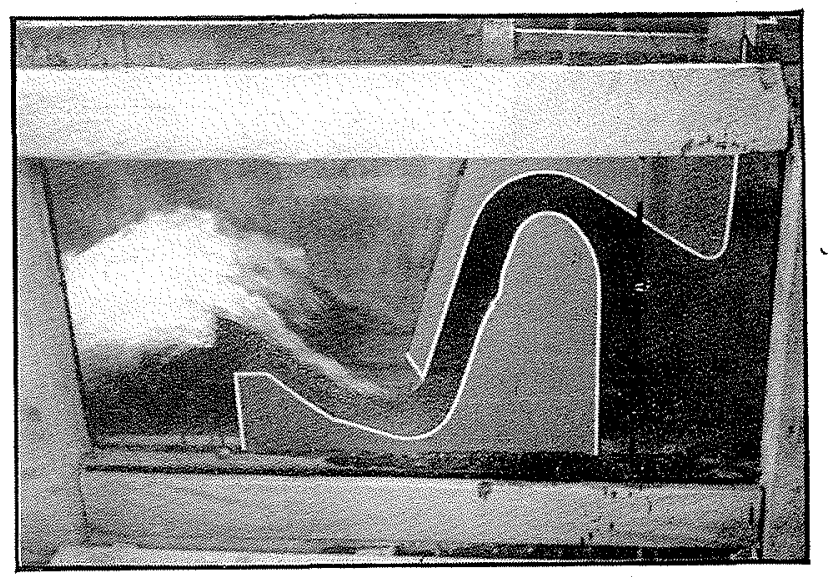

CHAMP-SUR-DRAC. - MODELE DU SIPHON

à-faux aux deux extrémités de l'arbre, qui repose sur les deux seuls paliers de l'alternateur. Le groupe ancien de $8.000 \mathrm{CV}$ a été conservé comme machine 
de secours. On concoit que, dans ce cas, c'est un remaniement très important de l'installation qui a été effectué puisque la puissance a été plus que quintuplée.

C'est au contraire moyennant des modifications relativement minimes qu'a eté modernisée la Centrale de champ-sur-Drac, également de lasociéte Génépale ae Force el Lumiere. Lancienne usine, qui comptait parmi les premièes installations de Frane puisqu'elle date de 1900, comportait à l'orisine cinc groupes centripètes de $1.350 \mathrm{CV}$ munis de distributeurs à tiroirs, utitisant sous $31 \mathrm{~m}$. un délìt de $25 \mathrm{~m}^{3} / \mathrm{sec}$ environ. Entre 1913 et 1918 , ces groupes firent place a six turbines francis, portant la puissance de $6.750 \mathrm{CV}$ à $7.000 \mathrm{CV}$ environ. en cas de coupure brusque du débit et peuvent fonctionner à débit partiel.)

- Les orifices de déversement de la cheminio d'équilibre furent modifiés de facon a permettr l'évacuation d'un débit de $12 \mathrm{~m}^{3} / \mathrm{sec}$.

Il ne pouvait être question d'installer dans l'ancien bâtiment le nouveau groupe Francis a axe vertical, de $9.430 \mathrm{CV}$ sous $28 \mathrm{~m}$. à $250 \mathrm{t} . / \mathrm{min}$. Alors que l'ancienne Centrale était toujours en service, une nouvelle usine fut construite à proximité immédiate. Ce n'est qu'au moment de son achèvement que fut eflectué, en un mois environ, le piquage de la nouvelle turbine sur la conduite, par une culotle de forme complexe, construite en béton armé. L'ancienne Centrale fut ensuite démontée et les 432



FONTPEDROUSE. - TURBINE EN ATELIER

Le réaménagement effectué entre 1940 et 1941 s'inscrit dans cet ancien cadre: la prise d'eau, le canal d'amenée, la conduite de $3 \mathrm{~m}$. 50 de diamètre, de 5 kms de long, en grande partie construite an béton, la cheminéc d'équilibre, furent intégralement conservés.

Le débit fut porté de 25 à $28 \mathrm{~m}^{3} / \mathrm{sec}$. grâce à deux modifications :

- Une batterie de siphons régulateurs de nivean, permit d'élever le plan d'eau dans le canal d'amenée, jusqu'à quelques centimètres des berges.

(Ces siphons, qui avaient été mis au point sur modèle réduit, dans les laboratoires des Ateliers N.B.P.P. sont conçus de façon à s'amorcer très rapidement, tomnes de ses machines (contre 286 tonnes seulement de matériel neuf) furent récupérées.

La turbine, du type Francis à axe vertical, a une vitesse spécifique relativement élevée (Ns 377). La bâche est construite en fonte, en quatre pièces. Malgré la puissance relativement modeste de la machine, ses dimensions sont assez imposantes du fait de la hauleur faible de chute.

Les conditions particulièrement défavorables dans lesquelles s'effeclue la régulation du groupe: conduite de $5 \mathrm{~km}$. de long, cheminée d'équilibre de section insuffisante pour maintenir la stabilité en réseau séparé, ont imposé l'installation d'un déchargeur de compensation qui est ici, en raison du débit élevé et de la faible hauteur de chute, un papillon 
de $1 \mathrm{~m} .75$ de diamètre, muni d'un double système d'équilibrage et d'un groupe de deux pompes à huile assurant sa refermeture lente.

La manouvre de Ia Centrale est semi-automatique : un seul agent y suffit, malgré la répartition des appareils sur les deux étages du bâtiment, srâce à un système de signalisation des incidents et d'arrêt automatique en cas d'avarie.

On peut se faire une idée đu gain réalisé grâce à ces nouveaux aménagements, relativement peu importants par rapport à l'ensemble des ouvrages, en notant les augmentations successives de puissance:

- De 5.000 CV à l'origine, la puissance est passée successivement à $7.000 \mathrm{CV}$ puis à $9.430 \mathrm{CV}$, cette dernière étape procurant une augmentation de $35 \%$ par rapport à l'équipement en service avant 1940 . En ce qui concerne la production, elle est passée de 35 à 47 millions de Kwh par an.

I'exemple de Champ-sur-Drac dont un seul homme peut assurer l'exploitation, nous incite a dire quelques mots du rôle de plus en plus importanif joué dans les Centrales par les systèmes de commarde automatique.

L'introduction de l'automatisme dans tous les domaines de lindustrie (depuis les trieurs de cigares jusqu'aux installations téléphoniques) est un signe caractéristique de notre époque. Dans le domaine des installations hydro-électriques, où la moindre défaillance peut avoir des conséquences graves, il importe d'examiner dans chaque cas avec une réserve prudente, les avantages et inconvénients d'un automatisme plus ou moins poussé.

Il est intéressant de remarquer, tout d'abord, que dans les centrales d'assez grande puissance il est toujours nécessaire de maintenir sur place du personnel d'entretien et, dans la plupart des cas, un chef de quart chargé de la mancuvre d'un poste d'interconnexion à départs multiples. Il serait alors illogique, dans de telles centrales, de réduire d presque rien le rôle de ce personnel dont la présence est par ailleurs indispensable. Le rôle véritable des systèmes automatiques est alors celui d'une centralisation des postes de mancuvre, de facon à faciliter la surveillance du chef de quart et lui permettre d'effectuer plus rapidement les mancuvres de démarrage et de modification de la charge.

On pent également remarquer à ce propos, que la sûreté de marche d'un équipement électro-mécanique automatique est d'autant plus grande que sa conception est plus simple : la surveillance d'un chef de quart « l'œil du maître » permet précisément d'éviter, dans une certaine mesure, les sécurités surabondantes, les « contrôles des contrôles » qui pouraient alourdir l'installation ef multiplier les contacts de ses relais électro-magnétiques.

Dans le domaine de la signalisation des incidents et du déclanchement automatique, des manouves d'arrêt en cas d'avarie, il semble que la question soit bien tranchée: moyennant l'installation de quelques détecteurs électro-mécaniques simples, mais dont la mise au point a d'ailleurs posé des problèmes techniques intéressants, moyennant la disposi-

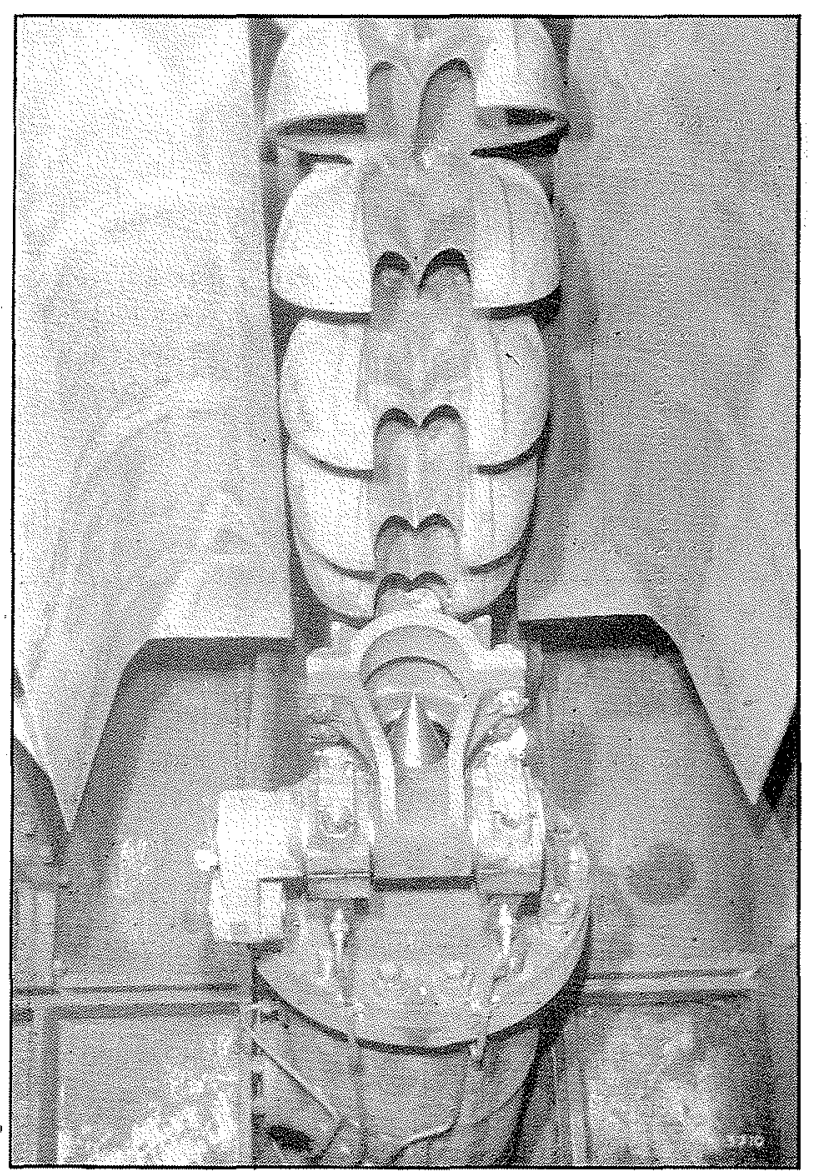

PONT-ESCOFFIER. - ROUE ET INJECTEUR

tion de servo-moteurs à huile ou à eau sous pression, judicieusement disposés, on peut accorder, tout bien pesé, aux systèmes électriques de sécurité une confiance plus grande qu'à l'altention et aux réflexes de l'homme de manœuvre le mieux entraîné.

Malgré les réserves faites plus haut, l'automatisme intégral, généralement réalisé sous forme d'un télépilotage, constitue cependant une solution intére;sante pour certaines Centrales de puissance assez faible et de manouvre simple, le plus souvent ne comportant qu'un seul groupe. C'est en particulier le cas pour des Centrales situées dans des vallées 
très reculées, comme cela se rencontre dans les Pyrénées. La Société exploilante dispose alors d'une équipe volante d'entretien, chargé de la surveillan-

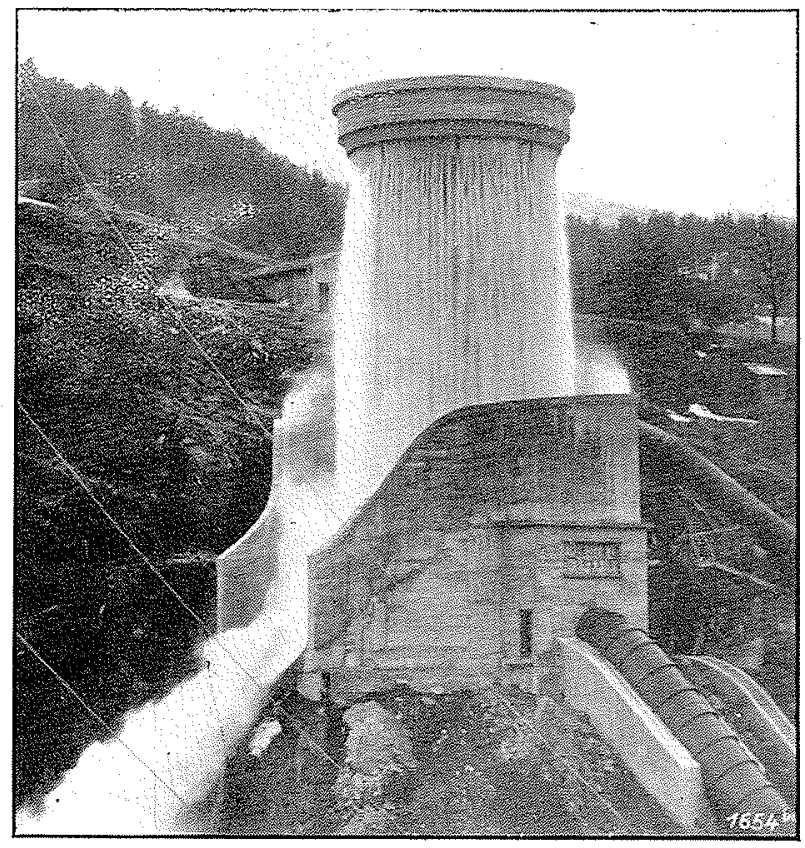

MONISTROL-D'ALLIER. - CHEMINEE D'EQUILIBRE

ce de plusieurs centrales, la manouve étanl assurée à distance, depuis une usine placée plus à l'aval et où vienment généralement converger les lignes éleetriques provenant des centrales satellites.
Alors que la commande manuelle directe n'aurait dé sowent possible que moyennant la construction d'un véritable villagge ef mème d'un réseau de téléphériques, comme dans les Usines de Tramezaigues et Lassoula dans les Pyrénées (n'oublions pas que le service continu requiert un personnel de quatre on cind hommes), la commande à distance réduit le personnel d'exploitation, soit à zéro, soit plus sottvent à un paysan, non spécialiste, chargé de la garde du bâtiment et éventuellement, appelé à effectuer de temps à autre des manouvres très simples.

De telles installations sont prévues, par exemple, tontes dans les Pyrénées, à Fontpédrouse (pilotage depuis la Centrale de la Cassagne, avec asservissement de la puissance au niveau amont), à Rioumajou, ou dans une gorge profonde, se monte actuellement un groupe Pelton vertical de $10.500 \mathrm{CV}$ sous $200 \mathrm{~m}$. de chute. Le pilotage sera effectué depuis la Centrale de Saint-Lary. De mome à Orgeix deux groupes Pelton seront pilotés depuis la centrale du Tét et enfin a Fabreges un groupe Francis de $11.100 \mathrm{CV}$ sous $105 \mathrm{~m}$. sera piloté depuis l'usine d'Artouste, distante de quelque 200 mètres.

Ces questions de manouve automatique nous conduisent à effeurer un problème qui dépasse de beaucoup le cadre de cet exposé, puisqu'il a trait aux réactions mutuelles des turbines et des réseaux interconnectés qu'elles alimentent: nous voulons faire allusion a la régulation des Centrales interconnectées et aux aspects multiples que revèt cette question complexe : répartition économique de la charge,

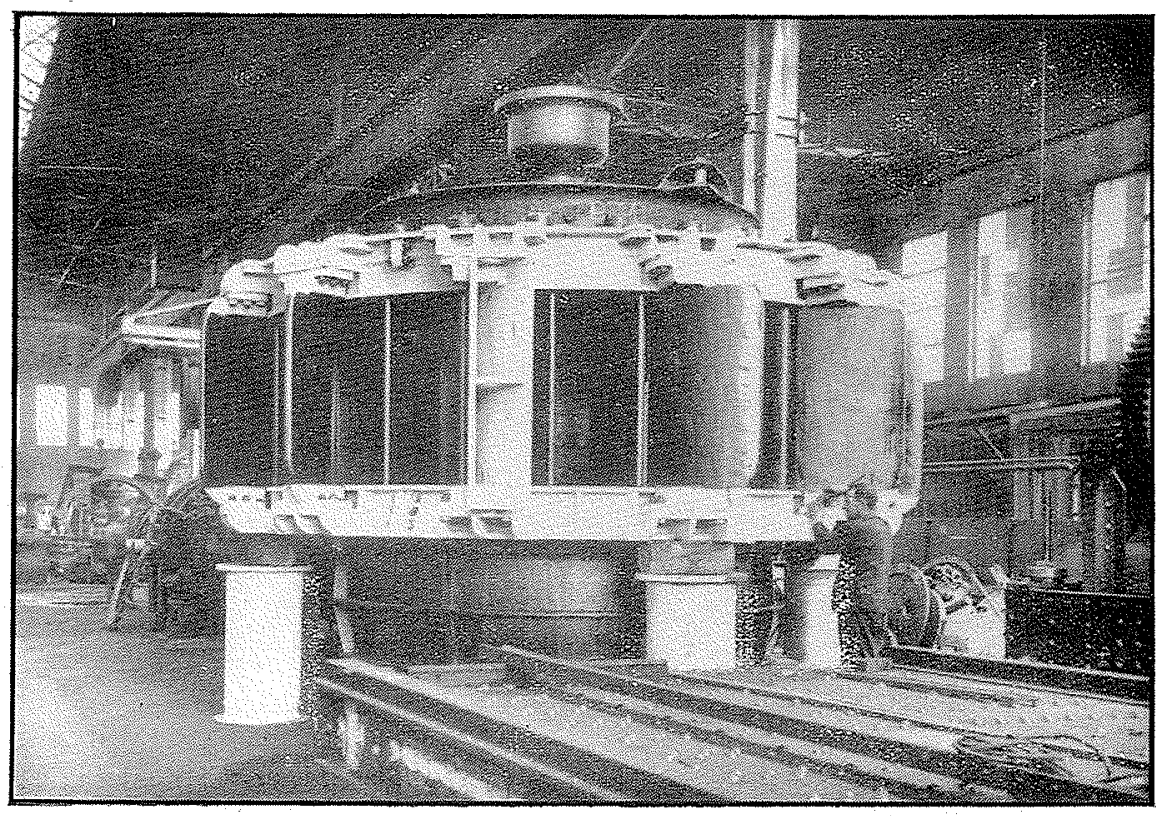

LABARRE, - DISTRIBUTEUR 
stabilité des groupes et des lignes d'interconnexion, réglage des puissances échangées et de la fréquenee.

Nous ne voulons pas parler ici des études el essais qui ont suivi les travaux de Mar. Fallou et Darrieus sur le réglage de fréquence-puissance ni discuter des incompatibilités entre le réglage rapide du lachymètre el l'action plus lenté d'un mécanisme automatique agissant sur le réglage de charge-vitesse th des réactions de ces systèmes sur la stabilité des lignes d'interconnexion et la reprise du synchronisme après un incident.

Nous voudrions simplement attirer l'attention sur un aspect assez simple de la question, qui avait été peut-ôtre un peu oublié depuis les tout premiers temps de l'interconnexion el sur lequel les Anglais titude de l'ordre de $2 \%$ sur la puissance; mais si le statisme est, non plus de $1 \%$ mais de 1 p. 1.000 , cette incertitude deviendra egale a $20 \%$.

Essayons maintenant, sur un grand réseau d'interconnexion, de régler la fréquence par le simple jen des tachymetres des régulateurs et proposons-nous de réaliser ce réglage avec une précision comparable à celle que montrent les meilleurs enregistreinents de fréquence réalisés vers 1939 , soit enviroi $1 / 20$ de période :

- Si fe réglage est eflectué, comme cela était couramment réalisé vers 1936, par une setile centrale "chef d'orchestre », le statisme de chacun des groupes de reglage deva etre inférieur a 1 \% : ces quelques groupes fonctionneront alors a charge cons-

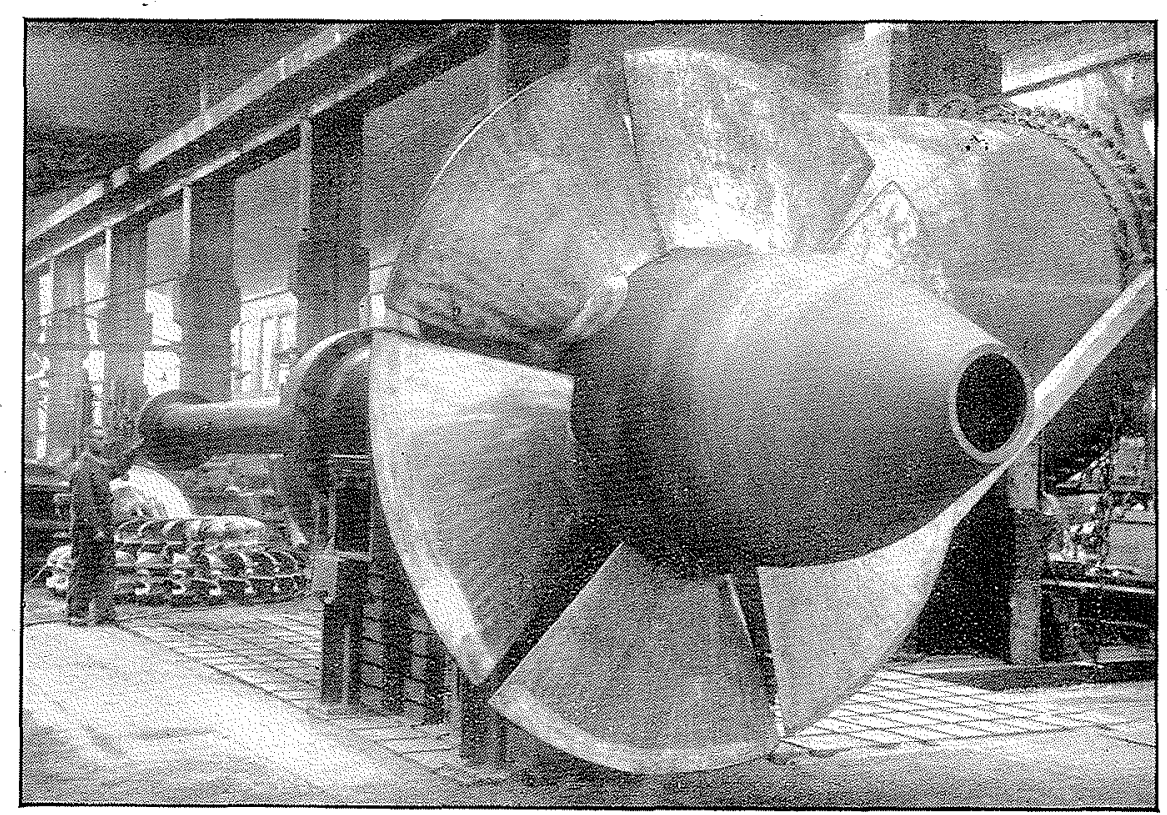

LABARRE. - ROUE-HELICE

et les Américains semblent avoir remis l'accent dans quelques articles et réalisations récents.

Du fait des jeux et du frottement de la timonerie des régulateurs, si bien construits soient-ils, l'ouverture du vannage qui correspond à une fréquence donnée n'est pas exactement déterminée : en fait, la puissance du groupe reste indéterminée entre deux valeurs, d'autant plus écartées l'une de l'autre que l'insensibilité du régulateur est plus grande, mais aussi que le décrément de statisme est plus petit.

Autrement dit, pour un groupe couplé sur un grand réseau, les imperfections du régulateur se font particulièrement sentir si le statisme est faible. C'est ainsi que sur un régulateur de précision, on pourrait associer, à un statisme de $1 \%$, une incer- tamment variable, le partage de puissance entre eux sera de plus, mal déterminé; le rendement moyen de l'installation sera alors peu élevé.

- On peut, au contraire, mettant bien entendu a part les centrales qui ne disposent d'aucune réserve hydraulique, faire participer au réglage un nombre importanl de groupes. Si, par exemple, pour aucun d'eux, la charge ne vient pas à varier de plus de $10 \%$, le même réglage de fréquence que plus haut sera obtenu gràce à un statisme de $1 \%$ et non plus de 1 p. 1.000 : le partage de la charge sera alors bien déterminé et le réglage sera effectué autom d'une position « de programme », dans une zone de bon rendement de chaque turbine.

Il semble donc que, en exploitant de facon assez 
complète les possibilités de réglage des centrales hydrauliques, on puisse obtenir un réglage précis de la fréquence, en même temps qu'une répartition bien déterminée de la charge, autour des valeurs "de programme » fixées par des consignes appropriées, ceci en faisant simplement appel an jeu des régulateurs tachymètres de grande précision.

Une conclusion serait actuellement prématurée, el l'on peut dire que la régulation des grands réseaux
Rappelons, en terminant, la contribution très importante apportée à l'avancement de ces problèmes, par les travaux ef discussions récents des $1^{\text {re }}$ et $4^{\text {th }}$ sections de la Société Francaise des Electriciens ot de Ieurs Présidents, MM. Langlois-Berthelot et Ailloret.

Revenons maintenant, apres cette excursion dans le domaine des grands réseatux d'interconnexion, aux turbines elles-mêmes.

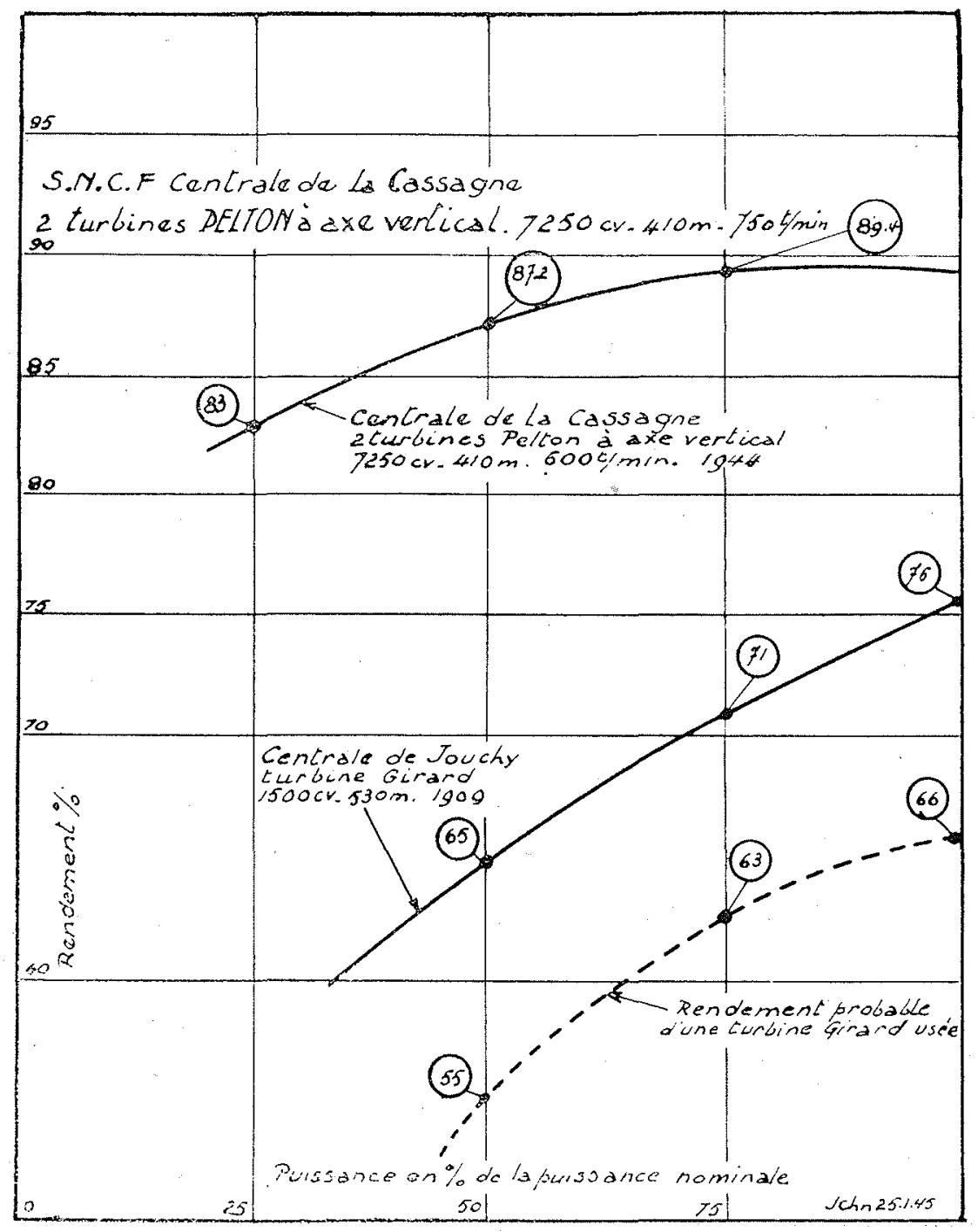

cherche encore sa voie, mais il n'est pas exclu de penser que celle-ci puisse etre trouvée dans le retour à un réglage très simple, tel que celui dont il vient d'etre question. Il va sans dire que, dans certains cas, il pourra être nécessaire de lui superposer in réglage correctif, qui permette en particulier d'éviter la surcharge momentanée des lignes d'interconnexion, ou de tenir compte de certains contrats d'échange à caractère impératif.
Nous allons examiner quelques particularités de machines réalisées récemment, ou en cours de construction, et se rattachant aux trois grandes familles: Pelton, Francis et Hélice.

Pour les unes comme pour les autres, une caractéristique essentielle réside dans la tendance vers des puissances unitaires de plus en plus élevées, vers des machines de dimensions de plus en plus imposantes. 
L'échelle sans cesse croissante des réseaux interconnectés, les moyens de construction de plus en plus puissants, tant en ce qui concerne le Génie Civil que les constructions mécaniques et electriques, reculent sans cesse les limites de puissances réalisables.

Si bien que nous en venons à l'idée que la limitation, peut-être la plus impérative, réside, dans ce domaine, dans le débit de nos fleuves et dans les hauteurs de chute dont l'équipement est possible dans des conditions convenables d'économie.

Si les $42.500 \mathrm{CV}$ de Brommat, les $52.000 \mathrm{CV}$ de Sarrans ont été, il y a maintenant quinze ans, des entreprises audacieuses, le nombre des machines de cette puissance est actuellement élevé dans le monde, en particulier en Amérique et les réalisations récentes, les plus caractéristiques nous mènent:

dans le domaine des turbines Pelton :

- aux 40.000 CV de Bissorte, 55.000 CV du Portillon, 56.000 CV d'Innertikithen en Suisse, et aux $85.000 \mathrm{CV}$ de Serra de Bubata au Brésil.

dans celui des turbines Hélice:

-- aux $60.800 \mathrm{CV}$ de Bonneville aux U.S.A., aux 65.000 CV de Hojum en Suède.

enfin, dans celui des Francis:

- aux 90.000 CV de Génissial, aux $100.000 \mathrm{CV}$ de Shipshaw, aux $116.000 \mathrm{CV}$ du BonlderDam, aux $135.000 \mathrm{CV}$ de Chastang, enfin anx 143.000 CV du Grand Coulee Dam aux U.S.A.

En ce qui concerne les dimensions des machines elles-mêmes, c'est avec les turbines Hélice de basse chute que les chiffres les plus imposants sont atteints.

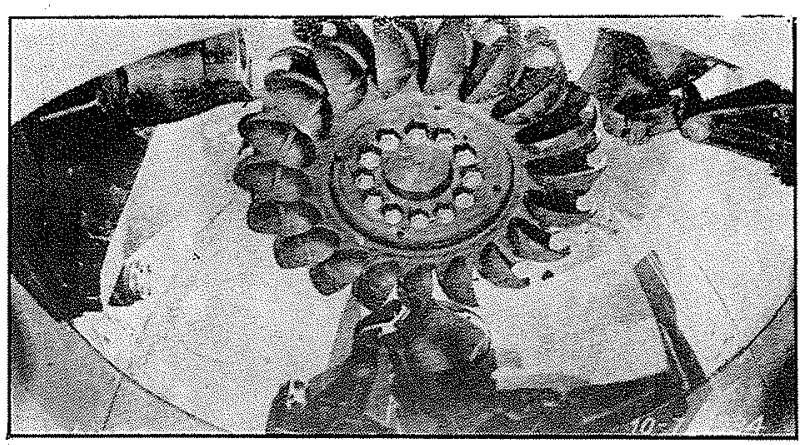

ESCALDES. - ROUE EN MONTAGE DANS LA CENTRALE

Les quelques $5 \mathrm{~m}$. des roues Francis de Génissial et du Grand Conlee ou les $5 \mathrm{~m} .80$ des 10 turbines de Beauharnois au Canada $(53.750 \mathrm{CV}$ sous $24 \mathrm{~m}$.) ne sont cependant pas très loin au-dessous des $6 \mathrm{~m} .80$ et $7 \mathrm{~m}$. 85 des roues Hélice d'Allbruck. Doggern et de Ryburg-Schworstaedt, en Suisse et enfin des $8 \mathrm{~m}$. des deux turbines suédoises de Vargon, les plus grandes roues du monde, de $18.000 \mathrm{CV}$ sous $4 \mathrm{~m}$. de chute, tournant a $50 \mathrm{t} / \mathrm{min}$. et absorbant chacune un débit de $312 \mathrm{~m}^{3} / \mathrm{sec}$. Peutêtre les centrales marémotrices, dont une étude poussée est en eo moment effectuée en France, nous conduiront-elles prochainement à des diamètres encore plus considérables, sans parler des turbines géantes que les Russes projetaient déjà en 1941, mais dont nous n'avons pas de nouvelle à I'heure actuelle.

C'est dans deux directions principales que, dans ce domaine des turbines Hélice à pales mobiles, les constructeurs orientent leurs recherches:

- D'une part, vers des turbines de relativement haute chute et de vitesse spécifique moyenne, qui jalonnent la zone de transition entre le domaine des roues Hélice et celui des roues Francis.

-. D'autre part, vers les turbines Hélice de basse chute et de grande puissance, pour lesquelles une vitesse spécifique élevée est généralement recherchée.

Rappelons brièvement, à cette occasion, que la vitesse spécifique, vitesse de rotation d'une turbin" semblable à celle projetée et développant 1 CV sous $1 \mathrm{~m}$. de chute, détermine dans une assez large mesure, la forme des roues de turbines. La vitesse spécifique est d'autant plus grande que la puissance ot la vitesse de rotation sont plus élevées, el que la hauteur de chute est plus basse.

On voit donc qu'une vitesse spécifique élevée per. met d'augmenter soit la puissance unitaire, soit la vitesse de rotation.

Parmi ces turbines Hélice de basse chute, citons les réalisations récentes c: :

- Swir, en U.R.S.S., avec quatre turbines di $37.500 \mathrm{CV}$ à $75 \mathrm{t} . / \mathrm{min}$. sous $11 \mathrm{~m}$., $\mathrm{ns}=725$.

- Pickwick Landing, aux U.S.A., avec deux groupes de $48.000 \mathrm{CV}$ sous $13 \mathrm{~m}$. à $82 \mathrm{t} / / \mathrm{min}$, ns $=724$.

- Reckingen, en Suisse, avec deux groupes de $24.000 \mathrm{CV}$, sous $8 \mathrm{~m}$.. 50 à $75 \mathrm{t}$. $/$ min., $\mathrm{ns}=765$.

- Jonage, dont les quatorze groupes de $6.000 \mathrm{CV}$ atteignent, en raison de leur vitesse de rotation particulièrement élevée $(214 \mathrm{t} . / \mathrm{min}$.) la vitesse spécifique de 760 et même de 900 sous les chutes les plus basses.

- Enfin, les turbines de Vargon, pour lesquelles une hanteur de chute de $4 \mathrm{~m}$. est associéc a une vitesse spécifique supérieure à 950 .

En France, où la prospection des basses chutes; n'est pas encore très avancée, et où les fleuves n'ont, malgré tout, pas la puissance de Russie ou d'Amé- 
rique, nous citerons outre Jonage, les quatre groupes de Pizancon sur l'Isère (14.600 CV sous $10 \mathrm{~m}$. 70), Kembs sur le Rhin (groupes de $36.600 \mathrm{CV}$ sous $16 \mathrm{~m} .50)$, les groupes, en cours de montage, de Labar'e sur l'Ariège $(6.440 \mathrm{CV}$ sous $11 \mathrm{~m}$. 50), de Cajarc sur le Lot $(6.200 \mathrm{CV}$ sous $9 \mathrm{~m}$.), enfin, parmi les projets, les trois groupes de Romans sur l'Isere et la Centrale de Donzère-Mondragon sur le Rhône.

Pour en revenir aux turbines Hélice de haule chute, nous remarquerons les installations récentes de :

- Harjavalta, en Finlande (47.000 CV sous $26 \mathrm{~m}$.). entre 150 et $350 \mathrm{~m}$, on peut hésiter, dans certains cas, à installer, soit des turbines Pelton à plusieurs jets, toumant assez lentement, soit au contraire des turbines Francis à faible vitesse spécifique, mais à vitesse de rotation élevée.

Si la solution Francis conduit souvent à une machine plus légère, la solution Pelton présente un srand intérêt en raison de son rendement élevé à charge partielle et des avantages qu'elle procure en ce qui concerne l'usure par une eau très chargée de sable.

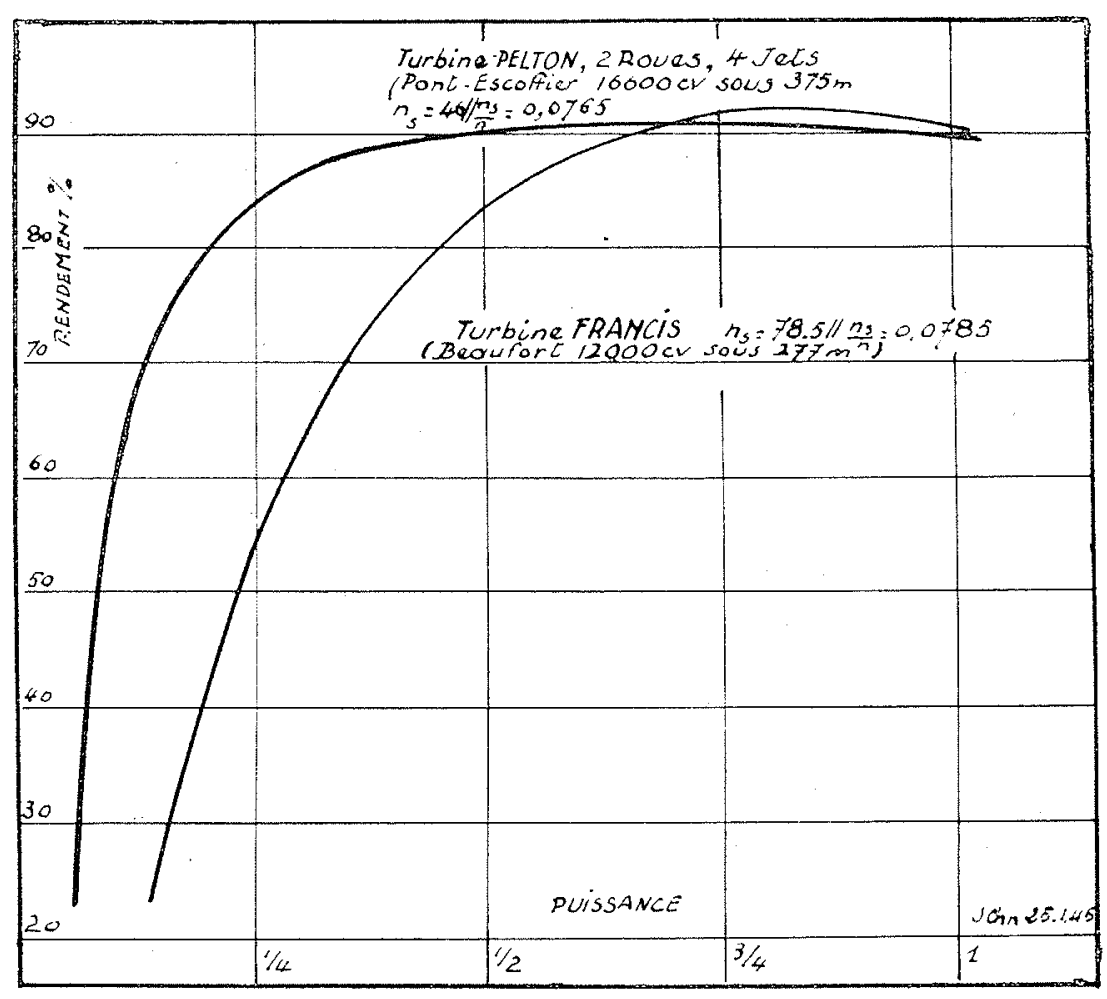

- Le Shannon, en Irlande (33.000 CV sous $32 \mathrm{~m}$. $50)$.

- Hojum, en Suède (65.000 CV sous $31 \mathrm{~m}$.).

- Enfin, en France, Cize-Bolozon (trois groupes de $10.750 \mathrm{CV}$ sous $17 \mathrm{~m}$. 50) et, an Maroc, les deux groupes d'Im'Font $(22.000 \mathrm{CV}$ sous $36 \mathrm{~m}$.).

A l'inverse des turbines Francis, à vitesse spécifique élevée, dont le rendement baisse très vite Jorsque l'ouverture diminue, ces turbines Hélice de haute chute permettent de conserver un rendement élevé, même aux ouvertures partielles.

C'est un avantage assez analogue que procurent, dans une autre zone de transition que nous allons maintenant examiner, les turbines Pelton de chute peu élevée par rapport aux turbines Francis de haute chute. Pour des chutes comprises, en gros,
Parmi ces installations « de transition 》 citons pour les Pelton :

- Fontpedrouse, dont nous avons déjà parlé sous $166 \mathrm{~m}$.

- Pont-Escoffier où quatre groupes de $16.600 \mathrm{CV}$ fonctionnent sous $375 \mathrm{~m}$. à $600 \mathrm{t}$. $/ \mathrm{min}$.

- Le projet d'Olette, sur la Tet, pour lequel la solution Pelton $(6.800$ CV sous $228 \mathrm{~m}$. a 600 t./ min.) l'a emporté, après une étude approfondie, sur une solution « Francis » a $1.000 \mathrm{t} . / \mathrm{min}$.

Parmi les Francis de haute chute, nous citerons entre autres:

- Le groupe de $12.000 \mathrm{CV}$ de Beaufort-sur-Doron sous $260 \mathrm{~m}$. de chute. 
- Les groupes de $42.500 \mathrm{CV}$ de Brommal, sous $255 \mathrm{~m}$.

- Les trois groupes d'Ixapantongo, au Mexique $(39.000 \mathrm{CV}$ sous $328 \mathrm{~m}$.).

Passons maintenant aux turbines Pelton et examinons, en nous référant à quelques machines-type, quelques dispositions caractéristiques.

En ce qui concerne les turbines Pelton à axe horizontal, les dispositions, qui comportent deux

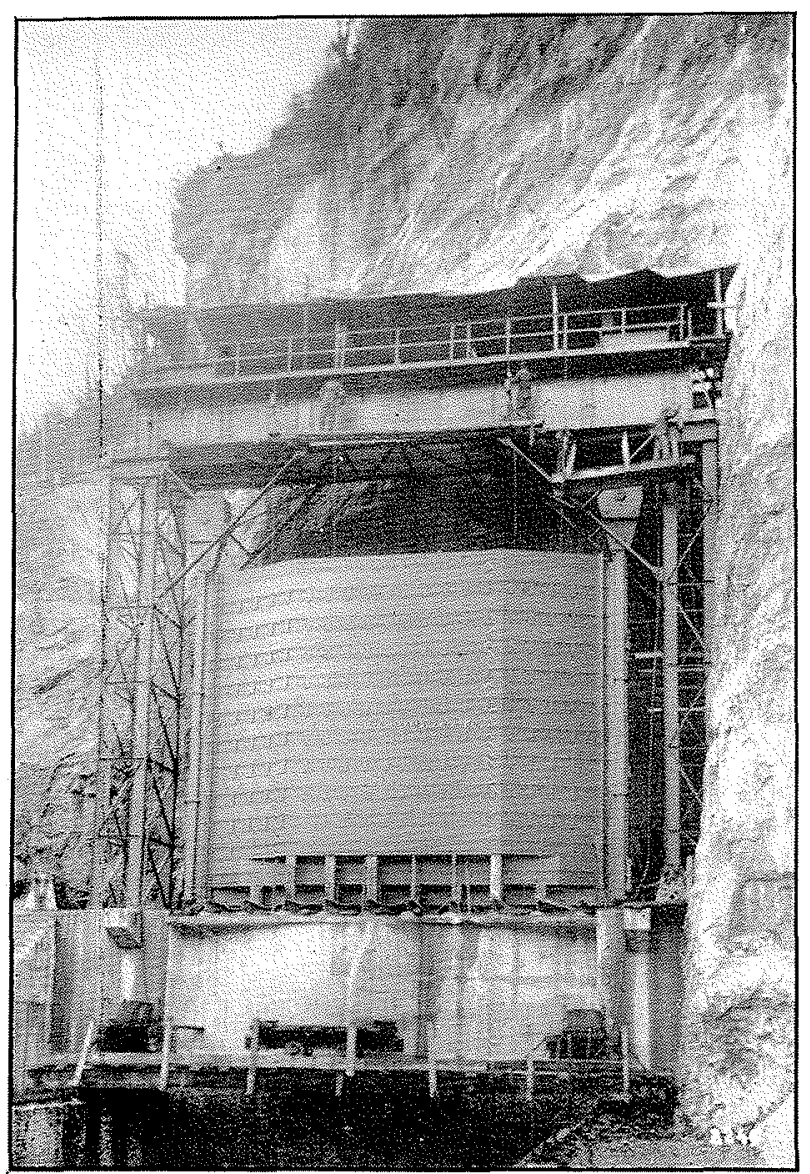

GENISSIAT. - VANNE DE DERIVATION

roues, done deux ou quatre jets, conviennent partculierement pour les chutes moyennes. Cependant, grâce à un tracé de roue à vitesse spécifique pen élevée (13 environ), la première disposition a été retenue pour les turbines de très hante chute de Fond-de-France $(1.000 \mathrm{~m}$.), du Portillon $(1.340 \mathrm{~m}$.) et de la Dixence $(42.500 \mathrm{CV}$ sous $1.750 \mathrm{~m}$.) la plus haute chute du monde.

Sous une chute de $1.160 \mathrm{~m}$. la disposition «Bissorte » a permis d'installer une roue unique de vitesse spécifique un peu supéricure (voisine de 17) dont le rendement est particulièrement élevé.

La disposition à axe vertical, qui a été retenue, par exemple pour les Centrales de la Cassagne et de Rioumajou, dont nous avons déjà parlé, permet de concentrer sur une roue unique les jets de deux, de trois et même de quatre injecteurs.

Ainsi, les cinq groupes d'Innertkirchen, en Suisse, à dewx jets, développent chacun $56.000 \mathrm{CV}$. sous $050 \mathrm{~m}$., cenx d'Escaldes, en Andorre, à trois jets, domnent $17.500 \mathrm{CV}$ sous $460 \mathrm{~m}$., celui de Rioumajou comporte quatre jets. Cette disposition nous a même permis, dans un avant-projet récent, de faire le croquis d'une machine dont les $118.000 \mathrm{CV}$ auraient été, grâce à quatre jets, fournis par un roue unique de $2 \mathrm{~m} .50$ environ de diamètre.

Il nous reste, maintenant, à donner un bref aperçu de quelques turbines Francis récentes, soit à axe horizontal, soit à axe vertical.

Parmi les turbines à axe horizontal, les plus intéressantes sont certes les turbines doubles:

- soit comportant deux turbines distinctes, une a chaque bout d'arbre de l'alternateur, comme au Sallet (c'est l'équivalent de la disposition Fond-de-. France en Pelton);

- soil comportant une roue double, avec un distributeur unique et deux coudes d'évacuation latíraux, comme cela a, par exemple, été fait récemment dans la Centrale de Morel, en Suisse Valaisanne, sur trois groupes de $24.000 \mathrm{CV}$ sous 263 mètres; de chute.

Les deux groupes Francis double du Péage-de-Vizille, dont la galerie a été altaquée en 1944 et dont la réalisation se poursuit, seront dans ce domaine les plus puissants du monde avec $35.000 \mathrm{CV}$ sous 132 mètres de chute.

Remarquons, à ce sujet, que pour des puissances importantes, seules les turbines doubles seront, en pratique, installées à axe horizontal. Pour les turbines Francis simples, la disposition à axe vertical est, daus la plupart des cas, la plus intéressante pour des machines de grandes dimensions : la bâche peut être plus facilement fondée, les démontages sont, en règle générale, plutôt plus faciles, et surtout la possibilité de placer la roue de la turbine à un niveau inférieur permet de réaliser des conditions d'aspiration plus favorables.

G'est donc parmi les turbines Francis simples à axe vertical que nous trouverons les machines les plus puissantes, dans de grandes centrales telles quo Cordéac, l'Aigle, Génissiat, ou celles de la Sungari, 
de Boulder-Dam, du Grand-Coulee-Dam, de Shasta, de Shipshaw.

Les hauteurs de chute sont, en gros, comprises entre 60 et 150 mètres :

- $64 \mathrm{~m} .50$ à Génissiat;

- $67 \mathrm{~m}$. à Shipshaw (Canada);

- $69 \mathrm{~m}$. à la Sungari (Mandchourie);

- $80 \mathrm{~m}$. à l'Aigle ; essentiels : le débit, la hauteur de chute, nn réser. voir d'accumulation de grande capacité.

En ce qui concerne plus particulièrement Ies turbines, leurs bâches spirales sont construites, soit sur place en tôle rivée, comme à Génissiat ou à la Sungari, soit en acier coulé, en plusieurs parties, comme à Cordéac, au Boulder ou à Grand-Coulee, soit encore comme à Saint-Etienne-Cantalès, en tôle rivée et

\section{Graphique des rendements comparés des turbines Girard et des turbines Pelton}

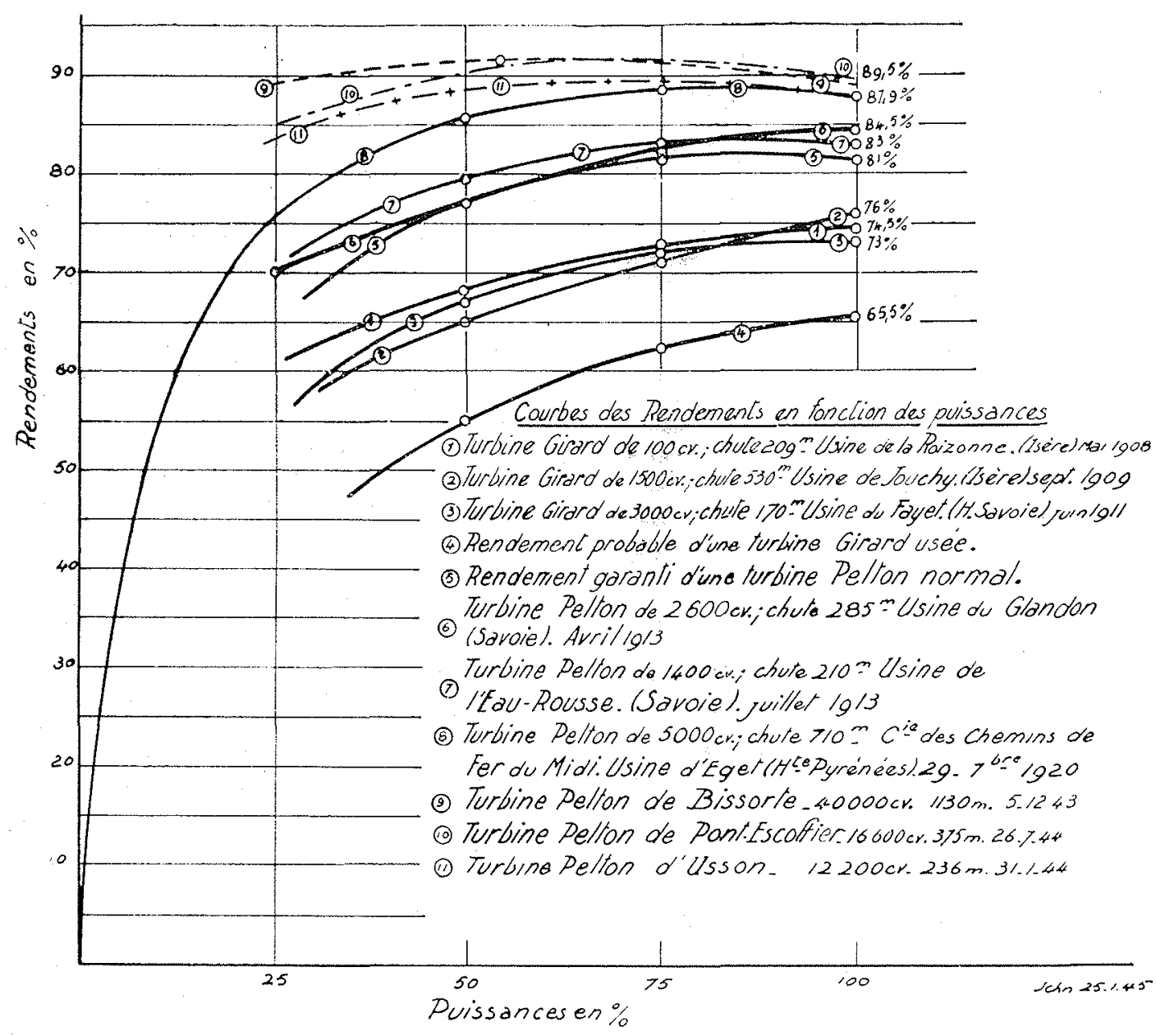

- 99 m. à Shasta (U.S.A.) ;

- 109 m. au Grand-Coulee-Dam (U.S.A.) ;

- $158 \mathrm{~m}$. au Boulder-Dam (U.S.A.).

C'est dire que ces centrales de grande puissance ne sont pas situées en haute montagne, où les bassins versants n'apporteraient pas un débit suffisant, ni en plaine, ou il serait difficile de créer une chute assez élevée, mais dans des régions de moyenne montagne, où il est possible de réunir trois éléments soudée, mais en plusieurs troncons construits à l'avance.

On concoit que la cuulée, l'usinage, l'assemblage, le transport de ces pièces énormes nécessitent la mise en cuvre de moyens d'une puissance inusitée. C'est ainsi que pour l'usinage et le montage des tur. bines de Cordéac (56.000 CV), de Génissiat $(90.000$ CV), et Chastang (135.000 CV), les Ateliers N.B.P.P., à Grenoble, ont construit un grand hall d'usinage et 
de montage, dans lequel un pont-roulant de 60-90 tonnes, placé att-dessus d'une fosse bétonnée, permet de monter ces machines en disposant d'une hauteur de crochet de plus de 15 mètres.

Pour donner une idée du poids et des dimensions de quelques pièces, voici des chifres:

- le cercle de vannage de Génissiat se démonte en quatre parties de 6 tonnes chacune;

- la rone, magnifique pièce d'acier coulé de quelques 5 mètres de diamètre (et c'est a $10 \mathrm{~cm}$. près environ qu'elle s'inscrit dans le gabarit des chemins de fer) pése 38 tomes;

- Ia bâche de Cordéac, $56.000 \mathrm{CV}$, en acier soudé, pèse plus de 100 tonnes et a èté usinée en six tranches, dont certaines dépassent 20 tonnes; maintenant un apercu de l'amélioration des rendements et des perfectionnements apportés au tracé des roues et aux méthodes d'essai qui permettent de juger, avant construction, des qualites d'une turbine nouvelle.

Deux graphiques comparalifs permettent de juger, quoique de facon assez imparfaite, des progres réalisés en ce qui concerne le rendement.

On sait que le rendement d'une turbine croît, dans une certaine mesure, avec les dimensions de la machine: ces graphiques montrent que les meilleurs rendements atteints en 1931-32, sur des turbines de grande puissance, sont égalés maintenant, dépassés même, par des turbines de puissance unitaire bien moindre. Il est intéressant de comparer,

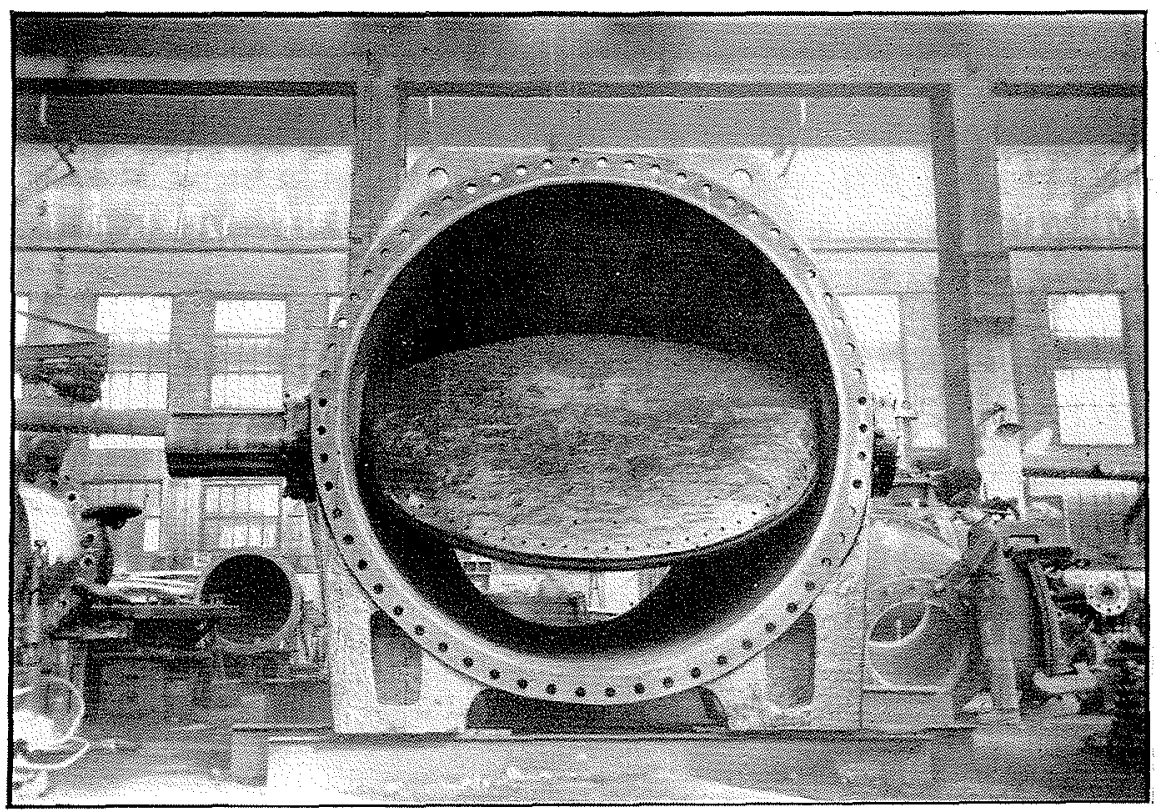

CORDEAC. - PAPILLON DE 2,350 M. MONTE

- la bâche du Boulder Dam pèse près de 200 tonnes, l'élément Ie plus lourd en pesant 36 .

En ce qui concerne Génissiat, la bâche pèsera dans les 300 tonnes, et l'on peut compter que, au total, les parties métalliques de la turbine pèseront un millier de tonnes.

Les papillons de garde, dont le corps et mème la lentille sont construits en plusieurs pieces, mesurant $3 \mathrm{~m} .50$ à Cordéac, $4 \mathrm{~m} .20$ au Boulder Dam, $5 \mathrm{~m} .20$ à Génissiat, $5 \mathrm{~m} .30$ à la Sungari. Quant aux turbines de $135.000 \mathrm{CV}$ de Chastang, sur la Dordogne, leurs papillons de garde auront $5 \mathrm{~m} .75$ de diamètre: c'est là une dimension record sous cette chute.

Nous venons de parler « dimensions ». Voici sur ce graphique, à côté des $80 \%$ des antiques turbines à tiroir, dont la première installation de Ghamp-sur-Drac est un exemple, les 84-88\% de la technique de 1919, avec les $92,8 \%$ de Sarrans (52.000 CV, 1931) presque atteints aujourd'hui par des machines beaucoup moins puissantes, telles que Ie Sautet $(1936,2 \times 8.000 \mathrm{CV}-92,5 \%)$ et même le Refrain $(1939,2 \times 3.140 \mathrm{CV}-90,7 \%)$.

Ces trois roues fonctionnent à des vitesses spécifiques comparables de l'ordre de $180 \mathrm{t}$. $\mathrm{min}$.

Sur l'autre graphique, relatif à des roues plus lentes (vitesses spécifiques de l'ordre de 120-130), on peut comparer les $90,6 \%$ de Brommat, avec $42.500 \mathrm{CV}$, aux $91 \%$ de la petite turbine de 4.000 CV de la Saussaz (1944). 
C'est grâce aux théories de la similitude, sur des roues modèle de petites dimensions, que l'on étudies le tracé des roues industrielles, et que l'on cherche à en parfaire le rendement. Une même roue d'essai peut, à difrérentes échelles, sous différentes chutes, a différentes vitesses, domner naissance a toute une gamme de turbines industrielles. La roue « Sautet 》, par exemple, a servi de modèle, entre autres : ont duré, en ce qui concerne le groupe AlsthomCharmilles-N.B.P.P. (1), de 1939 à 1941 et ont comporté le relevé de plus de 65 diagrammes en collines, ce qui correspond, en gros, à 9.000 points d'essai.

Cette méthode d'essai, suivant la similitude classique dite de Combes-Ratean, n'est cependant pas complète et ne rend pas compte, en particulier, des phénomènes de cavitation.

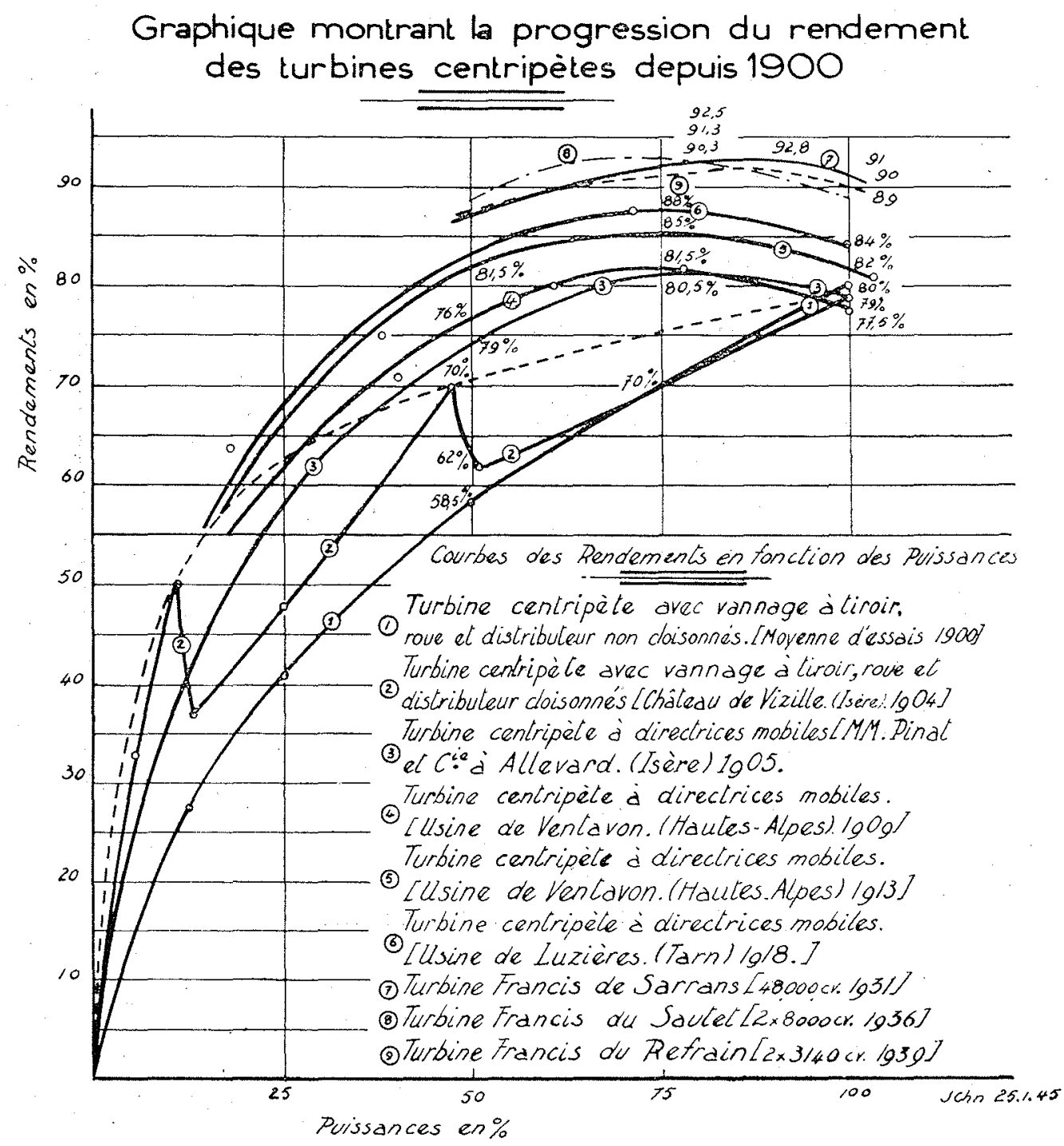

- à l'échelle 1/2, aux turbines du Sautet et du Refrain ;

- à l'échelle 1/3, à la turbine de $28.000 \mathrm{CV}$ de Cordéac;

— à l'échelle $1 / 5$, à la turbine de $56.000 \mathrm{CV}$ de Cordéac.

Les essais sur modèle réduit, qui ont permis de mettre au point les tracés de la roue de Génissiat, de sa bâche, de son distributeur, de son aspirateur,
Nous n'insisterons pas sur les graves inconvénients bien connus auxquels peut donner lieu la cavitation des turbines hydrauliques: baisse de rendement, vibrations, usure. D'autre part, l'installation d'une turbine est, dans certains cas, d'autant

(1) Sur les quatre turbines cie Génissiat actuellement en construction ou en montage, deux sont de construction Alsthom-Char truction ou en montage, deux sont de construction Alsthom-Char Fives-Lille. 
moins onéreuse qu'on approche de plus près sa limite de cavilation. Il est done extrêmement intéressant de savoir, par la reproduction sur modèle réduit des régimes de cavitation, a partir de quelle puissance, de quelle chute, de quelle hauteur d'aspiration une turbine risque de caviter.

Plusieurs méthodes ont été pour cela proposées. La plus perfectionnée d'entre elles a été mise en de la baisse de rendement, les limites entre lesquelles la lurbine industrielle pourra fonctionner sans danger de cavitation.

Cet exposé aura montré, nous l'espérons, d'une part, la complexité des problènes qui sont posés chague jour aux constructeurs hydrauliciens, d'autre part, la grande diversite des types, des formes,

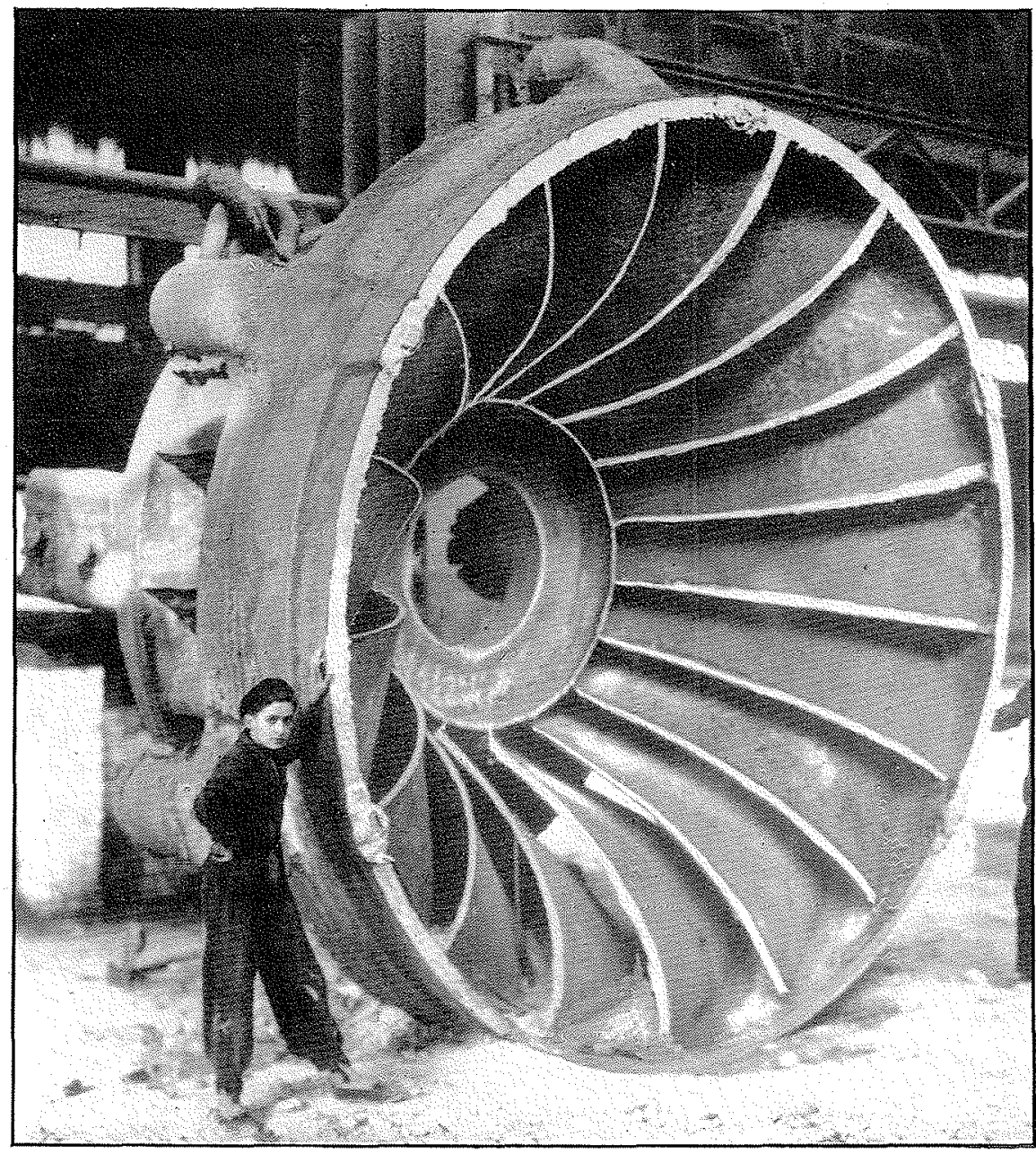

GENISSIAT, - ROUE DE TURBINE FRANCIS

œuvre aux Ateliers N.B.P.P., à Beauvert, sur la demande de la Compagnie Nationale du Rhône, pour les essais de la roue destince à la Centrale de Génissiat. Dans cette station d'essais, établie à $12 \mathrm{~m}$. au-dessus du sol, une rone réduite a été essayée sous $7 \mathrm{~m}$. de chute, dans un circuit fermé dont une pompe de $200 \mathrm{CV}$ entretient le débit, et sous une dépression importante, qui peut atteindre $9 \mathrm{~m} .40$ de vide. Les essais, déjà terminés depuis deux ans, ont permis de préciser à la fois par écoute des vibrations consécutives à la cavitation et par mesure des dimensions des machines qu'ils doivent conce. voir et réaliser.

Quelle commune mesure pourraiton trouver, en effet, entre une petite turbine Pelton de quelquos, dizaines de CV et une turbine géante telle que celle de Génissiat?

Devant des réalisations aussi hardies que celles que nous venons de passer en revue - et qui pourtant, si l'on examine de près la question, sont le fruit d'une lente évolution - devant celles qui, demain peut-être, seront à l'échelle non plus du 
Rhône ou du Colorado, mais du Volga, du Congo ou de l'Amazone (les Russes ne parlent-ils pas de turbines hélice de $14 \mathrm{~m}$. de diamètre, et les usines a marées n'imposerontelles pas davanlage encore), on peut se demander, avec quelque inquictude, si lhomme ne se trouvera pas, un jour, completement dépassé par son cuve. Que devient, autrement dit, la personnalite de I'homme, le respect qui lui est dû, quand il doit participer à des tâches si gigantesques, que seul, un travail entièrement discipline, qui doivent faire appel, avant tout, à son intelligence?

Nous n'en sommes heureusement pas encore là, el l'on peut dire que des réalisations de très grande envergure, telles que l'Aigle, Génissiat ou Chastang font encore plus appel à l'labilaté manuelle du tourneur ou de l'aléseur, à la science de l'ingénieur, a leur esprit de discipline personnelle, qu'à l'embrigadement dans une masse où se perdraient les persomnalités.

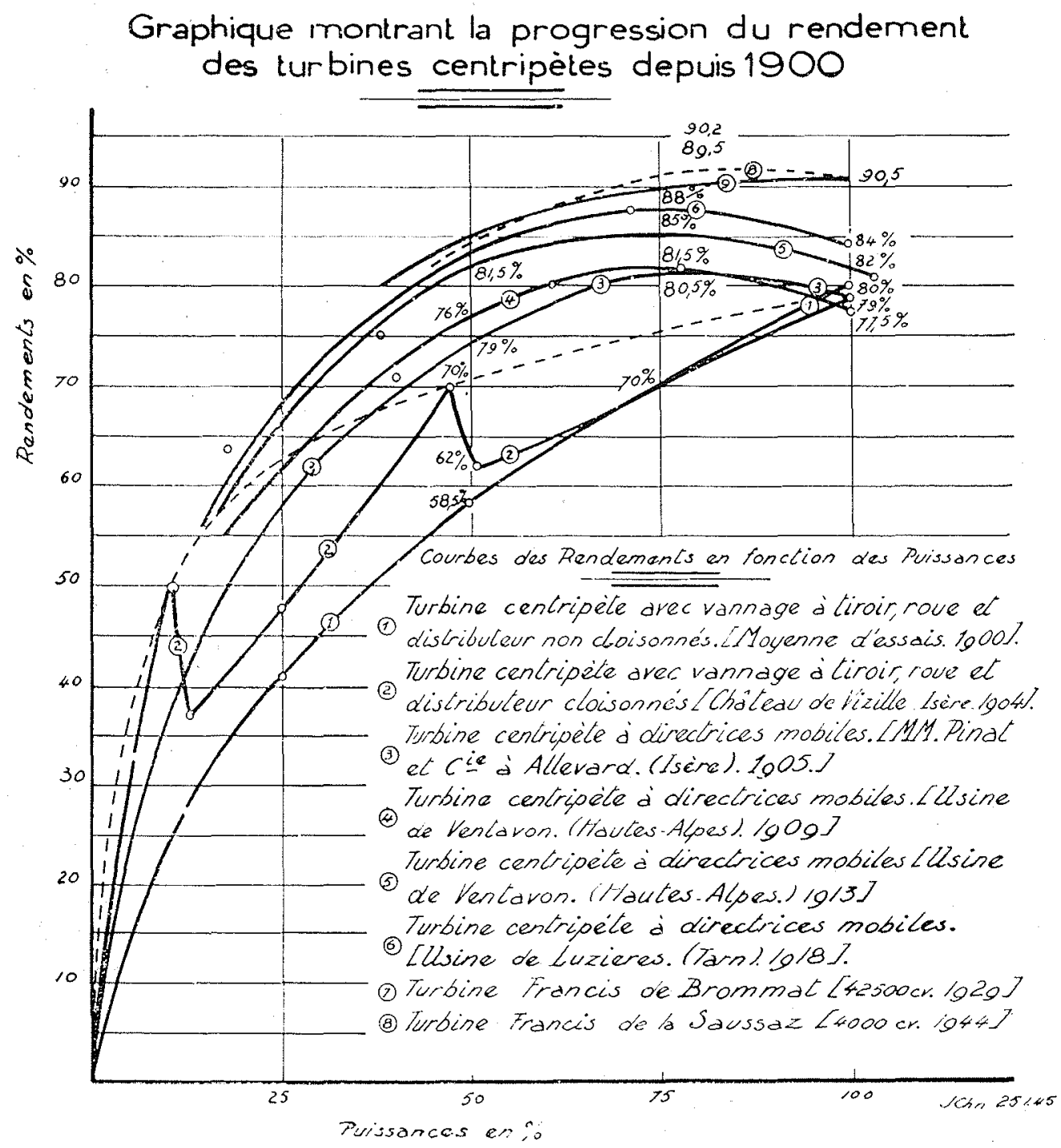

pour lle pas dire un jour asservi, permet de coordonner les activités de quelque 10.000 ou 50.000 ouvriers, contremaîtres et ingénieurs?

Nous avons vu tout à l'heure que les conditions géographiques pouvaient apporter une limitation à la puissance des turbines. Une autre frontiere, plus étroite peut-être, ne pourrait-elle pas résulter de l'homme lui-même et des limites qu'il convient peut-être d'assigner en toute raison, à des travaux
Alors que nous avons pu, au cours des quatre dernières années, nous demander parfois, aux temps les plus sombres de l'occupation, si la France ne portait pas en elle les germes d'une décadence irrémédiable et n'était pas capable de déborder le cadre étroit de l'action individuelle, le fait qu'il nous ai été possible, en pleine tourmente, de mener à bien des tâches aussi difficiles, est pour nous le meilleur gage de confiance. 